\title{
Physical and technological analysis of the AIGaN-based UVC-LED: an extended discussion focused on cubic phase as an alternative for surface disinfection
}

\author{
H. I. Solís-Cisneros ${ }^{a}$, C. A. Hernández-Gutiérrez ${ }^{a}$, A.-Rehman Anwar $^{b}$, P. Y. Sevilla-Camacho ${ }^{c}$, J. L. Camas-Anzueto $^{a}$, \\ R. Grajales-Coutiño ${ }^{a}$, R. Trejo-Hernández ${ }^{d}$, Y. L. Casallas-Moreno ${ }^{e}$, and M. López-López ${ }^{f}$ \\ ${ }^{a}$ Tecnológico Nacional de México/Instituto Tecnológico de Tuxtla Gutiérrez, Optomechatronics Group. \\ ${ }^{b}$ Laboratory of Nitride Semiconductor Physics, Institute of High-Pressure Physics, Polish Academy of Sciences. \\ ${ }^{c}$ Cuerpo Académico de Energía y sustentabilidad, Universidad Politécnica de Chiapas. \\ ${ }^{d}$ Programa de Nanociencias y Nanotecnología, CINVESTAV-IPN. \\ ${ }^{e}$ CONACYT-UPIITA, Instituto Politécnico Nacional. \\ ${ }^{f}$ Departamento de Física, CINVESTAV-IPN
}

Received 16 November 2021; accepted 11 December 2021

\begin{abstract}
Crisis in coronavirus times requires understanding the effects on society and establishing efficient mechanisms to prevent infections. The disinfection of personal protection equipment by UVC light remains a key opportunity area. Therefore, this letter presents the main drawbacks and challenges on the fabrication of deep ultraviolet LEDs based on III-nitrides, such as the substrate selection, dislocation reduction, the increase of external quantum efficiency, enhancement of the radiative recombination in the active region, the complications to reach high Al content in AlGaN-based UVC LED avoiding the reduction of the p-doping, replacing the p-GaN contact layer by $\mathrm{p}$-AlGaN without hindering the deposition of ohmic contacts. Furthermore, the cubic phase is suggested as a promising candidate for AlGaN UVC-LEDs applications as is discussed in this work.
\end{abstract}

Keywords: Ultraviolet light-emitting diodes; disinfection; external quantum efficiency; AlGaN; cubic.

DOI: https://doi.org/10.31349/RevMexFis.68.020301

\section{Introduction}

At of the time of writing, more than 250 million people have been infected and more than 5 million deaths have been reported since the pandemic related to new Severe Acute Respiratory Syndrome Coronavirus (SARS-CoV-2) began [1]; however, the problem is more serious than it seems. More than 37 million HIV patients and their treatment are at risk [2], just like the attention for noncommunicable diseases such as hypertension, and cardiovascular emergencies, diabetes and its related complications, even cancer patients [3-5] which were partially or completely diminished to dedicate the best efforts as possible to Coronavirus Disease named COVID-19. This situation has been triggered by the lack of staff strongly related to workers' reassignment, medicine shortage, and a decrease in public transport services [6].

From another perspective, stay-at-home policy and social distancing have brought consequences in mental health such as depression and anxiety [7,8], even changing the perception of safety and security. Only the North America and Russia-Eurasia regions ( 2 out of 9 regions in the world) became more peaceful in comparison with 2019. This means that shocks like COVID-19 and the resulting recession have affected education, societal safety, security, as well as the domestic economy, especially in the more vulnerable and conflicting regions [9].

According to a report from the Institute of Economy and Peace, global violence has cost around USD 14,528.9 billion in Purchasing Parity Power terms in 2019 [10]. Thus, the fol- lowing scenario is not promising while violence continues to increase. Moreover, at least 88 million people could fall into extreme poverty at the end of 2020 and up to 35 million more in 2021, indicating a three-year reversal of world prosperity and fight against poverty [10]. This increase in poverty is largely due to the fact that most of the people who work in the informal sector without social security have been affected by mobility restrictions, social distancing, and lockdowns [10].

When people worldwide began to need some protection to continue their ordinary life during the SARS-CoV2 outbreak, some products got more expensive and inevitably inaccessible. At this point, also the World Health Organization (WHO) published recommendations about what barriers and personal protection equipment were useful to diminish the number of deaths and seriously ills [11]. These recommendations provoked the facemask and respirators market into an increment from USD 1.03 billion (before the outbreak) to more than USD 2.5 billion at the end of 2020 , reaching up to USD 2.8 billion in 2026, depending on the pandemic evolution [12], and the overprices that, in some cases, have reached a factor of five [13]. As a consequence, both the production and the pollution related to these have increased up to more than 20 times from February to June 2020 [14].

To meet the demand and overprices, elaboration of homemade masks and reuse of standardized ones have been taken as two options; however, the reuse of equipment exposed to pathogens may cause a considerable increment in confirmed cases by reduction of effectiveness [15]. A review of diverse disinfection approaches points to UV radiation as a useful 
and effective disinfection method to face the SARS-CoV-2 spread and the COVID-19 contagion. Fighting the pandemic by a disinfection approach will contribute to reduce social distancing and restart activities such as commerce, tourism, and education, which will counteract the side effects on mental health, security, and the economy. On the other side, facemasks costs might be reduced while the demand for disposable Personal protective Equipment (PPE) is reduced from the reliable reuse of disposable medical gowns, facemasks, and respirators.

Despite Light Emitting Diode (LED) technology being closely related to electronic devices development in different areas such as lighting [16], communications, and entertainment, a growing interest is showing up in healthcare to generate faster and more accurate tools (e.g., medical equipment, vital signs monitoring devices, disinfection, sensors) $[17,18]$. Due to the recent health crisis in the world, special efforts on disinfection research have been made to obtain and optimize barrier mechanisms avoiding infectious agents like SARSCoV-2 [19], MERS-CoV [20], and Ebola [21]. Although Low-Pressure Mercury (LPM) lamps and, still in development, UVC LED devices (200-280 nm) are effective against viruses and bacteria [22], material research and deposition techniques are widely studied because of deoxyribonucleic acid (DNA) and ribonucleic acid (RNA) destructive properties of UVC radiation [19, 23, 24]. Is important to mention that LPM lamps have characteristics that put them at disadvantage compared to UVC LEDs (e.g., big size, fragile glass components, usage of $\mathrm{Hg}$, and shorter lifetime). However, it is still necessary that LEDs increase their power levels and enhance their efficiency in order to reduce exposure time achieving the same disinfection effectiveness [23, 25]. One of the most important issues to deal with is the fact that, as the wavelength emission shortens, the device shows less power [26]. Therefore, is essential to determine the optimal wavelength for the device design $[26,27]$ considering short exposure times, since as the exposition time increases the UV disinfection could be counterproductive [28]. Fortunately, UVC-LEDs are still being improved and efficiency is expected to increase so that this technology substitutes the LPM lamps for UV disinfection. In order to unfold the challenges and drawbacks in the development of UVC-LED based on III-nitrides, it is important to approach the fact that disinfection requires UVC range emissions to inactivate viruses.

\section{Virus inactivation mechanism}

The spread of diseases and the capability of human beings to endure infections are susceptible to the amount of infectious load to which we are regularly exposed. It is remarkable to mention that different environments refer us to different viruses and bacteria loads [29], which inevitably puts human life at risk for disease contagion. Viruses depend on a host to maintain activity and reproduce; thus, they have to adhere to a cell that allows them to continue transmitting their genetic information. This is encoded as a sequence of nucleotides, which are made up of nitrogenous bases linked to deoxyribose or ribose in DNA or RNA, respectively [30]. The nitrogenous bases that are part of DNA are Guanine-CytosineAdenine-Thymine, while in RNA Uracil replaces Thymine. Using the machinery of the cell, the nucleic acid (DNA or RNA) of the virus produces proteins through a mechanism that involves messenger RNA (mRNA). These proteins function as antigens that stimulate an immune response in the body [31,32]. The mRNA is decoded and used for designing vaccines to face the COVID-19 pandemic [33]; however, the challenge of delivering the artificial mRNA to the cells (either naked mRNA in vivo or wrapping it with lipid-based carriers) remains [34]. While pharmaceutics and researchers are looking to diminish both contagion and disease symptoms, it is also necessary to consider different approaches (inactivated or attenuated virus, replication vectors, RNA or DNA, among others) [35] and the side effects (from fatigue, pain, chills and skin reactions to fever, urinary retention, and even moderate Bell's palsy) 31 to guarantee vaccine safety [36]. On the other hand, RNA-based viruses show rapid mutation compared to DNA-based viruses [37]; thus, SARS-CoV-2 mutations result in more infectious virus strains that could complicate the vaccine development and efficiency [38,39].

\section{$\mathrm{UV}$ radiation for disinfection}

While the development of vaccines and treatment against SARS-CoV-2 and COVID-19 continues its progress [36], it is reliable to aim at disinfection as a mechanism to prevent contagion [28]. In contrast with vaccine effectivity, UV disinfection is not affected by virus mutations due to the fact that the effectiveness of UVC devices on coronaviruses inactivation is based on the formation of dimers by adjacent uracil or thymine present in RNA or DNA, respectively. This genetic disruption mechanism is triggered by photoreactions and is due to the sensitivity of these nitrogenous bases to UVC radiation [40,41]. First, the photons emitted around $260 \mathrm{~nm}$

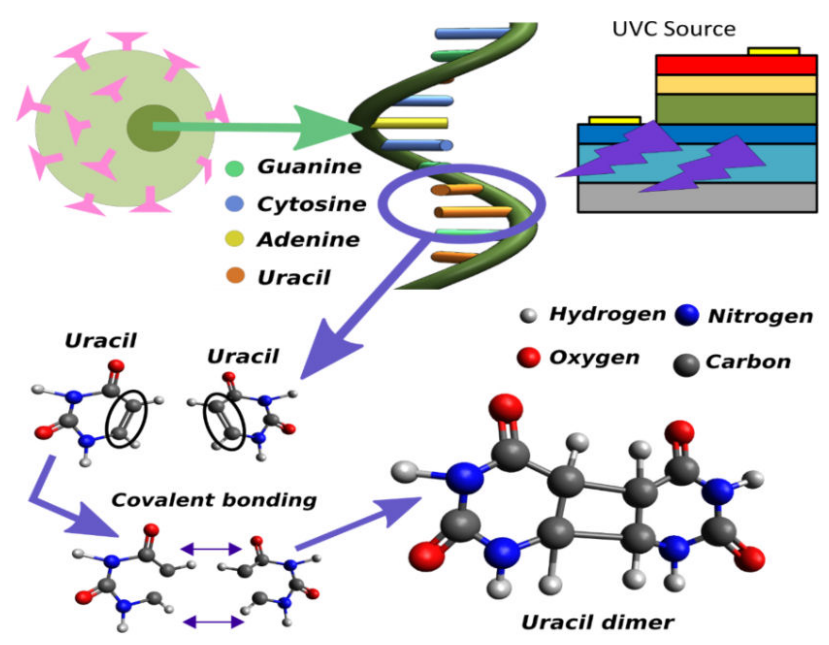

FIGURE 1. Mechanism of virus inactivation by uracil dimer formation. 
are absorbed by the nitrogen bases breaking a specific carbon covalent bond in the ring of the molecule (as shown in Fig. 1) so, at this moment, the two adjacent bases are linked through stronger covalent bonds provoking genetic mutation by chain disruptions [22,40,41]. Because of this RNA (DNA) sequence's fractures, the capabilities of viruses and bacteria are depleted, preventing their lodging, reproduction, and the infection that causes to the host [42]. Therefore, the use of germicidal UVC radiation devices to disinfect potentially infectious surfaces is taken as an important object of study to compare efficiency, risks, and benefits between technologies $[27,43,44]$. It is possible to disinfect surfaces such as walls and furniture inside hospital rooms [45], even N95 masks and PPE achieving a $90 \%$ inactivation of viruses, making possible their reuse [46]. Nevertheless, it is crucial to determine the correct dose with minimal damage to filtering facepiece respirators [47], considering that the longer wavelength is, the higher the used dose must be [26]. Some complementary treatments or disinfections techniques include both UVA and UVC radiation to minimize the risk of contagion. First, UVA $(315-400 \mathrm{~nm})$ is used for the reactivation of viruses and bacteria which sequences had been disrupted. Then, a UVC stage is in charge of destroying the most microorganisms as possible by dimerization of thymine or uracil; whatever the case, the experiments are typically performed with Medium Pressure and Low-Pressure Mercury lamps as UVA and UVC sources, respectively [48], increasing the risks of poisoning due to accidental contact with $\mathrm{Hg}$. In the first instance LPM, as the main source of UVC light, has been replaced by its analog LED. Although LEDs emit in narrower bandwidths, these can be set in principle to any wavelength depending on the material.

UV LEDs have their origins in Gallium Nitride $(\mathrm{GaN})$ [49], which allows them to be grown as crystal thin films, reducing dimensions compared to other light sources such as incandescent bulbs or fluorescent lamps. However, these LEDs are limited by the wavelengths available in GaN structures (over $350 \mathrm{~nm}$ ). In this case, it leaves aside the idea of using it in UVC development. Therefore, the incorporation of aluminum ( $\mathrm{Al})$ to attain shorter wavelengths and cover almost the entire UVC range, has been investigated by using aluminum-gallium nitride (AlGaN) alloys [50].

\section{AlGaN-based UV LED}

III-N semiconductors are widely studied for LED fabrication, from gallium nitride $(\mathrm{GaN})$ to ternary alloys such as aluminum-gallium nitride ( $\mathrm{AlGaN})$ and indium-gallium nitride (InGaN) [51-55]. All these materials have different optical, morphological, and electrical properties which extend their use to different fields. The hexagonal (h) structure is the stable phase of III-nitrides, but the cubic (c) phase (metastable) has also been studied due to distinct properties that we will discuss below. In order to produce a lightemitting device in the UVC range, the energy gap $\left(E_{g}\right)$ of the material must emit photons with energy between 4.43 and

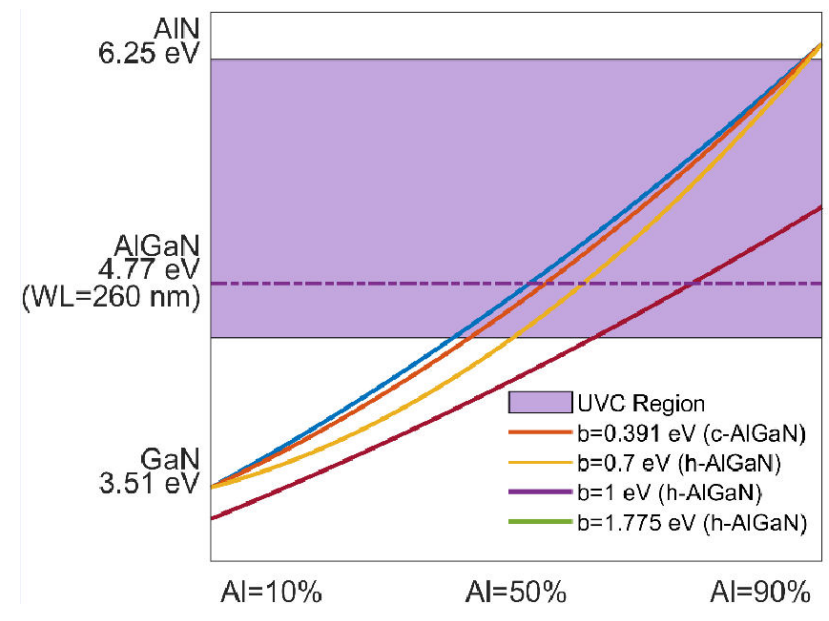

FIGURE 2. Effect of the bowing parameter on the Energy gap of AlGaN LED.

$6.20 \mathrm{eV}$. Thus, from the fact that the range of the AlGaN energy gap extends from 3.27 and $3.47 \mathrm{eV} \mathrm{[56]} \mathrm{(for} \mathrm{c-GaN} \mathrm{and}$ h-GaN, respectively) to 5.7 and $6.25 \mathrm{eV} \mathrm{[56]} \mathrm{(for} \mathrm{c-AlN} \mathrm{and}$ h-AlN, respectively), and that the bandgap is directly related to $\mathrm{Al}$ content [57], $E_{g}$ can be varied by increasing (or decreasing) the $\mathrm{Al}$ content according to Eq. (1) [58].

$$
E_{\mathrm{Al}_{x} \mathrm{Ga}_{1-x} \mathrm{~N}}=x E_{\mathrm{AlN}}+(1-x) E_{\mathrm{GaN}}-b x(1-x) .
$$

To estimate the alloy's $E_{g}$, for the hexagonal phase, it is important to mention that despite early works point to $b=1$ $\mathrm{eV}$ as a correct value [59], the bowing parameter was also taken as $b=0.7 \mathrm{eV}$ to reduce deviations [60]. However, from more recent work, b in Eq. (1) can be set as $1.775 \mathrm{eV}$ for hexagonal phase, and $0.391 \mathrm{eV}$ for cubic phase [56]. Fig. 2 illustrates the change of $E_{g}$ as a function of $\mathrm{Al}$, it also shows how the bowing parameter affects the expected Eg of the AlGaN alloy. Furthermore, it is important to mention that LEDs are evaluated by their External Quantum Efficiency (EQE), which involves Internal Quantum Efficiency (IQE), Light Extraction Efficiency (LEE), and Carrier Injection Efficiency (CIE), which are related through the Eq. (2) [61].

$$
E Q E=I Q E \times L E E \times C I E .
$$

In order to obtain high-power and efficient UVC LED, three general aspects from Eq. (2) should be considered: first, to enhance the CIE by increasing carrier injection to the active region $[62,63]$ and preventing the carrier leakage $[64,65]$. Second, in order to reach higher IQE, it is necessary to diminish the dislocations present in III-N semiconductor $[66,67]$ and, in the case of the hexagonal phase, reduce the effects of strong piezoelectric and spontaneous polarization [68]. And third, the LEE is increased by employing different approaches from patterned substrates to photonic crystals [69-72]. In all cases, it is essential to consider that, while wavelength increases, i.e., from 267 to $297 \mathrm{~nm}$, this shifting provokes an increase in UVC doses required for disinfection of around $26 \mathrm{~mJ} / \mathrm{cm}^{2}$ [26]. 


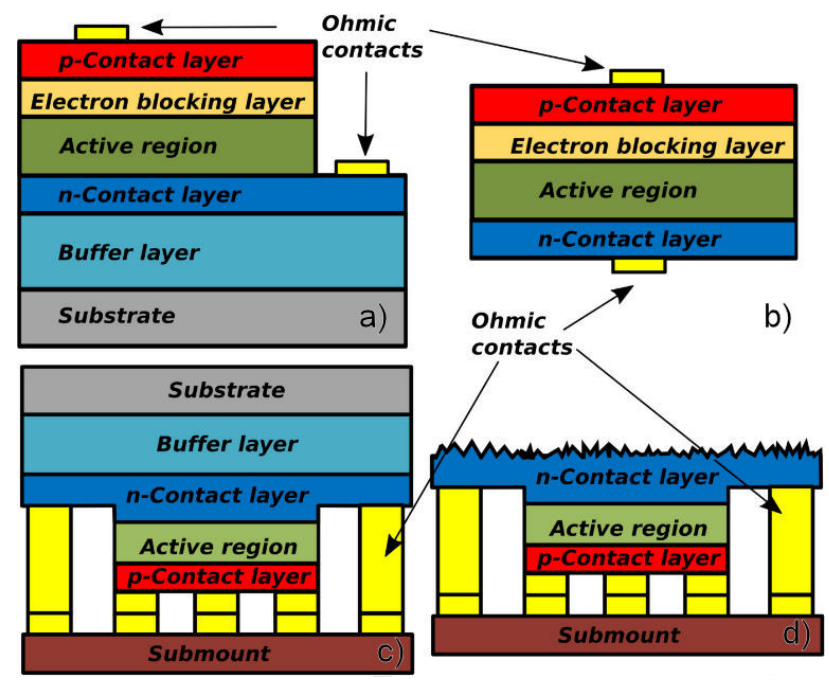

FIGURE 3. Structures of the UVC LEDs: a) Typical lateral structure, b) Vertical structure or V-chip structure, c) Flip-Chip structure, and d) Thin Film Flip-Chip Structure.

\section{LED structure considerations}

Recently, the use of $\mathrm{GaN}$ as the contact layer remains in UVC LEDs design, mostly due to some considerations about doping and transparency that will be mentioned in more detail in later sections. However, the p-contact layer is not the only stage in UVC LED development that could be designed from different approaches. There are superlattices as strain absorber [73] and extra layers [74] that benefit the carrier injection without sacrificing the carrier densities, even carrier blocking layers [75-77] with different structures to prevent the leakage [78].

The typically used LED structures are shown in Fig. 3 in order to visualize the different stages of LED development. In general, the design starts with the substrate and its corresponding buffer layer, the n-type contact layer, the active region, the electron blocking layer, and the p-type contact layer. However, sometimes additional p-type layer is grown to avoid unwanted effects of the p-AlGaN contact layer [79]. Each layer carries diverse challenges [52]: the substrate and its buffer must provide the least number of defects, be as transparent as possible, and allow heat extraction. The ntype layer must spread the current efficiently and manage the strain; the active region faces the main task of increase IQE and carrier confinement which allows emitting photons by energy quantization, and the p-type layer, must enhance the CIE. Thus, it is needed a low resistance in both layers and contacts.

\section{Substrate and buffer layer}

The first element to consider is the substrate, here it is very important to choose the appropriate crystal structure since there are relevant differences between the cubic and hexagonal phases of III-N materials [56]. It is important to note that the maximum concentration of $\mathrm{Al}$ that the cubic phase allows before the material shifts from direct to indirect bandgap is around $70 \%$ [56]. This is a severe drawback because when a semiconductor changes to an indirect bandgap, the probability of radiative recombination is reduced because a phonon is necessary to conserve momentum in the emission of a photon [80]. On the other hand, the hexagonal phase of AlGaN presents the advantage over the cubic phase of requiring a lower concentration of $\mathrm{Al}$ to reach higher $E_{g}$; nonetheless, when working with h-AlGaN, it is very important to consider that built-in internal electric fields cause a large shift to lessenergetic emission [80], as will be discussed in the next sections. In general, due to the fact that the hexagonal structure is the stable phase of III-nitrides, the state of art unfolds studies that support sapphire as the main substrate in UVC LED growth [74,81-87].

Even though, it is important to consider that crystalline sapphire or silicon carbide films (SiC) still suffer from excessive defects from substrate-film mismatches as thickness increases [88]. This is provoked, to a large extent, by the different lattice constants between the alloy components (AlN, $\mathrm{InN}$, and GaN) [57,89-91] and the change in the target $\mathrm{Al}$ content throughout the multilayer [92]. Moreover, due to its absorption, $\mathrm{SiC}$ is not explored enough [93], although, in order to face this drawback, UVC LED on SiC could be processed into a thin-film flip-chip to obtain high LEE [93].

During the growing procedure, these defects introduce nonradiative recombination centers in the semiconductor, which contribute significantly to the reduction of LED efficiency [94]. In other words, the radiative recombination, which is required to emit photons, is limited by dislocations or defects in each layer during their growth affecting both the EQE and the Light-Output Power (LOP) as shown in Figs. 4a y $4 \mathrm{~b}$, respectively. Thus, dislocation densities of $10^{9} \mathrm{~cm}^{-2}$ are needed to reach $50 \%$ or higher IQE [95]. The state of art reports that nanopattern sapphire substrates show a $98 \%$ increase in EQE by reducing the number of dislocations compared to flat sapphire substrates [96]. Another consideration is required since III-V alloy components have different lattice constants; therefore, an AlN buffer layer must be grown to replicate the substrate structure in order to avoid cracks and reduce dislocations [97]. The following layer on the hexagonal AlN must be designed to improve the LED technology and its efficiency [98].

Furthermore, superlattice structures (SL) on sapphire substrates are employed in order to decrease the dislocations and prevent cracks as the thickness increases by reducing the tensile strain [99]; these SLs filter the dislocations significantly $[73,100]$. Besides, the use of AlN/GaN SLs has shown intense luminescence in the deep ultraviolet region from 4.4 to $5.0 \mathrm{eV}$ (related to the $\mathrm{GaN}$ layer thickness) when an AlN/GaN SL $(1.8 \mathrm{~nm}, 0.6 \mathrm{~nm}$, and $0.75 \mathrm{~nm})$ is grown by $\mathrm{MBE}$ on a $4 \mathrm{H}-\mathrm{SiC}$ substrate [101]. Moreover, an AlGaNbased transition layer might be grown after the AlN buffer layer which results in a crack-free smooth epitaxial film with threading dislocations around $3.8 \times 10^{9} \mathrm{~cm}^{-2}$ on a Si sub- 


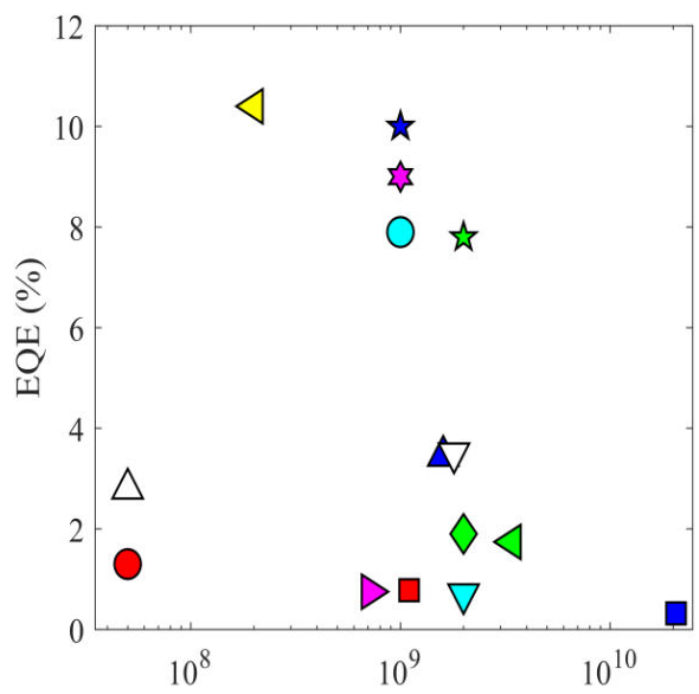

a)

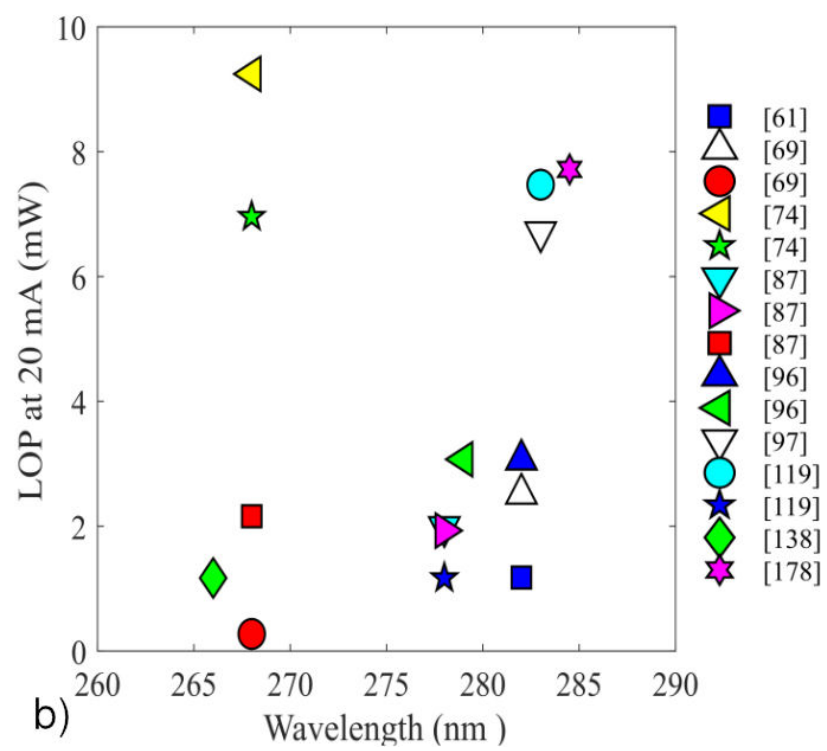

FIGURE 4. Dislocation effect on LED performance a) Relationship between dislocation density and EQE at $20 \mathrm{~mA}$ and b) LOP as a function of wavelength.

strate achieving a roughness of $1.5 \mathrm{~nm}$ which is critical to obtain highly efficient quantum wells (QWs) [102].

\section{The challenge of enhancing the active region}

Once the substrate has been chosen and buffered, the next step is to study the photoluminescence emission peak, which is directly related to the active region. Here, it is necessary to mention that light emission characteristics can strongly be modified by using different quantum heterostructures such as quantum dots (QD) [103-105], nanowires, and quantum wells $(\mathrm{QW})$ confined between quantum barriers $(\mathrm{QB})$.

Moreover, the efficiency in the active region is closely related to three different types of recombination mechanisms, as shown in Eq. (3), employing the ABC model [106].

$$
R=A n+B n^{2}+C n^{3}+f(n),
$$

where $R$ is the recombination rate, $n$ the carrier concentration, and $f(n)$ the leakage current as a function of the carrier concentration. A, B, and C represent the Shockley-Read-Hall (SRH), radiative, and Auger recombination coefficients, respectively. These three coefficients refer to the recombination mechanisms illustrated in Fig. 5. First, radiative (or band-toband) recombination is the base of photoemission in the active region and involves electrons from the conduction band, which recombine with holes in the valence band, releasing energy as photons during the process. Secondly, SRH recombination plays an important role when high levels of dislocations are present in the structure, generating heat [106]. Finally, Auger recombination is related to the carrier concentration and allows that a third carrier absorbs the energy released by electron-hole recombination, raising it higher in the conduction band or pushing down a hole deeper in the valence band [107].

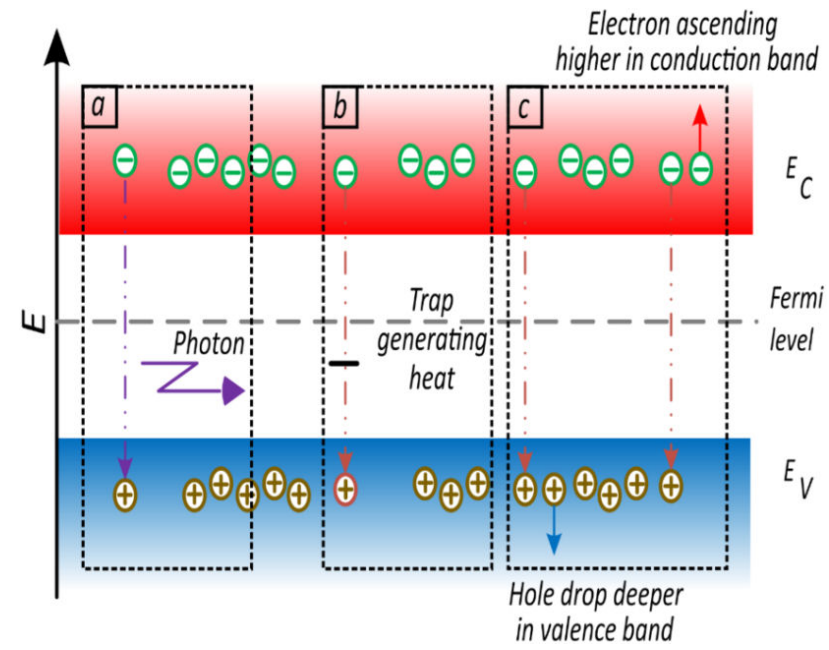

FIGURE 5. Recombination mechanisms, a) radiative recombination, b) Shockley-Read-Hall recombination and c) Auger recombination.

For the optimization of device performance i.e., radiative recombination, the selection of the thickness of the QWs and barriers is crucial. In the hexagonal phase, due to the presence of the spontaneous and piezoelectric fields, the electrons and holes inside the QWs are separated, leading to a decrease in the probability of radiative recombination, as illustrated in Fig. 6a), as well as a shift of the emission toward lower energies. This is known as the quantum-confined Stark effect (QCS) and its influence is shown in Fig. 7a), where the emission wavelength is plotted as a function of QW width (red color line) and compared with the emission without internal electric fields (blue color line) for $\mathrm{A}_{l 0.61} \mathrm{Ga}_{0.49} \mathrm{~N}$ QWs sandwiched between AlN QBs. For reference, the theoretical data are also compared with experimental results from Haughn 

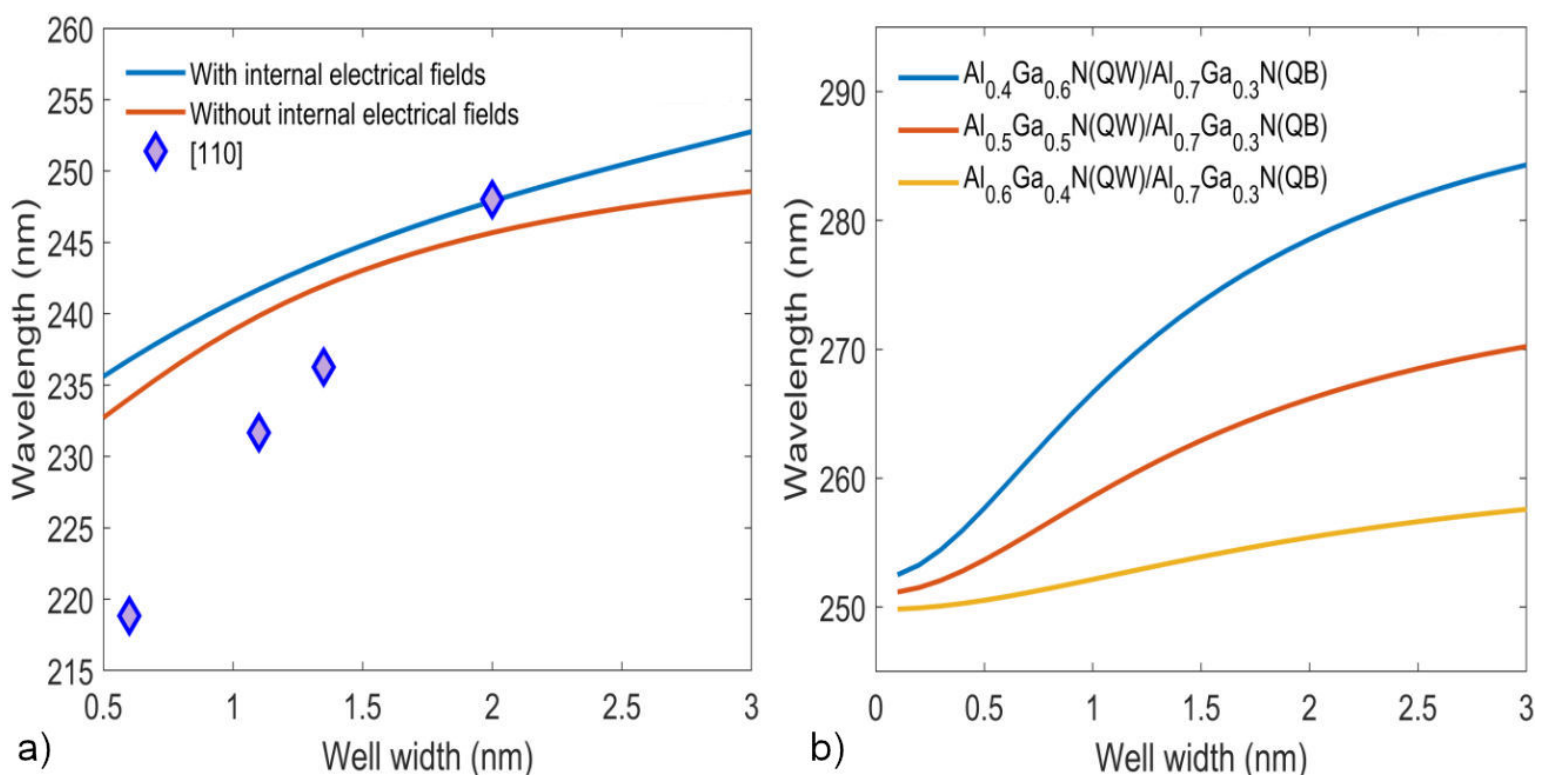

FIGURE 6. QW width effect on emission wavelength of $\mathrm{AlGaN}$-based UVC using QW/QB structure based on a) $\mathrm{Al}_{0.61} \mathrm{Ga}_{0.49} \mathrm{~N} / \mathrm{AlN}$ in hexagonal phase, and b) built-in electric field as function of $\mathrm{Al}$ mole fraction and thickness (insert) in the barrier. The emission wavelength was calculated using the ground levels in the QWs by solving Schrödinger's equation. The energy shifts were obtained as described by Phys. Rev. B, 28 (1983) 3241. The built-in electric fields were calculated according to Morkoç, "Handbook of Nitride Semiconductors and Devices", vol. 1. Wiley-VCH, 2009.

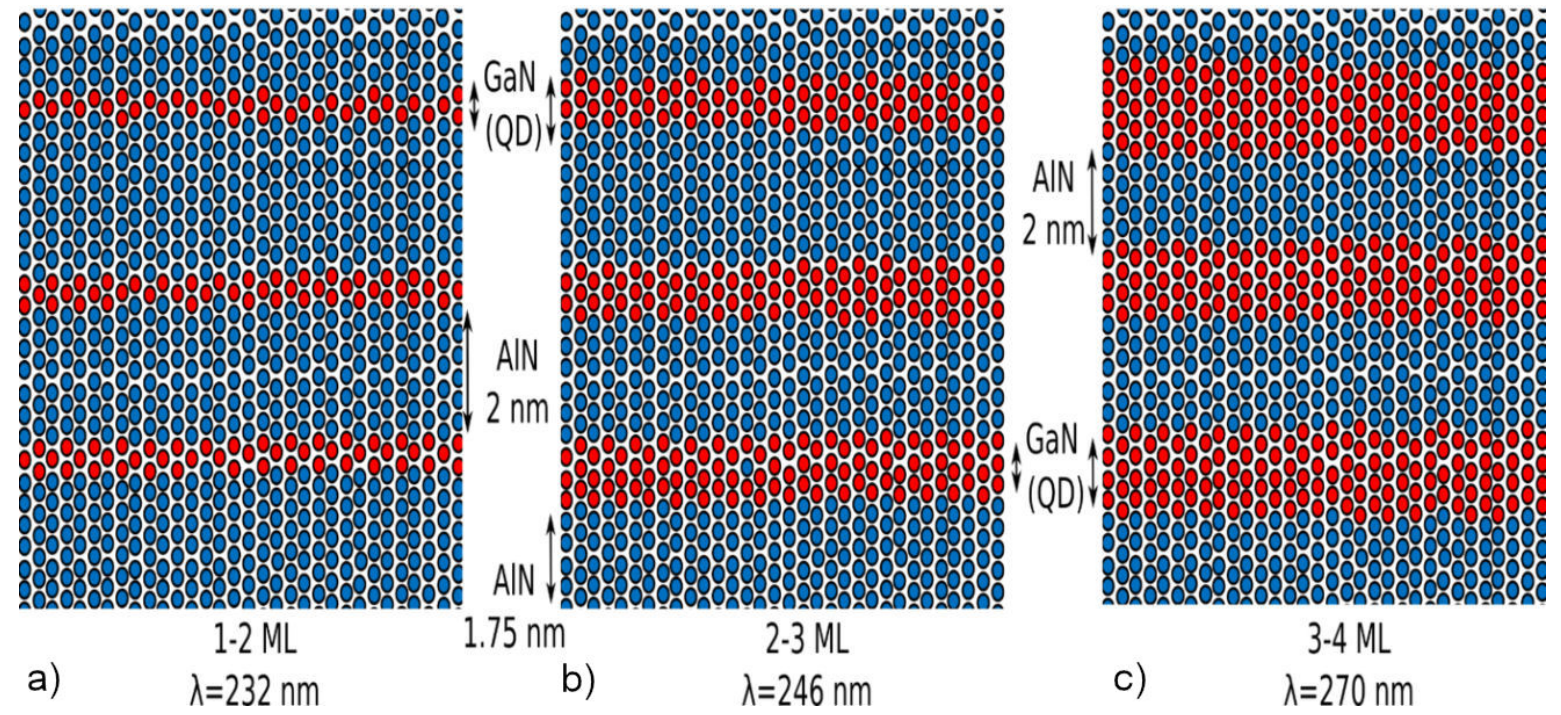

FIGURE 7. QD scheme from Islam et al119 with emission at a) $232 \mathrm{~nm}$ (1-2 ML QDs), b) $246 \mathrm{~nm}$ (2-3 ML QDs) and c) $270 \mathrm{~nm}$ (3-4 ML QDs).

et al. [108]. As observed in Fig. 7a), the red-shift of the emission wavelength increases with both the QW thickness and strength of the built-in electric field. Here, it is important to remark that the internal electric field strength is on the order of $\mathrm{MV} / \mathrm{cm}^{-1}$ and rises with the $\mathrm{Al}$ content and thickness in the barriers, as presented by the theoretical curves in Fig. 7b) and its inset. In this case, the calculation of the electric field includes both spontaneous and piezoelectric polarization and is consistent with previous similar studies [109-111]. These theoretical results show that the QCS effect has a large influence on the QW transition energies, reducing the IQE.
Thus, in order to improve the radiative efficiency in hexagonal AlGaN-based UVC LED, very thin QWs have to be employed so that the QCS effect is mitigated (Fig. 6b)), and the oscillator strength of radiative transitions are increased [80, 112, 113]. For example, Hirayama et al. have investigated the effect of QW thickness on the LOP [114]. They proposed a device structure with $1.3 \mathrm{~nm}$ thick QW with remarkable improvement in radiative performance. Therefore, combinations of three aforementioned parameters, i.e., well width, barrier thickness, and $\mathrm{Al}$ content, could be engineered to minimize the influence of the QCS effect in opto- 

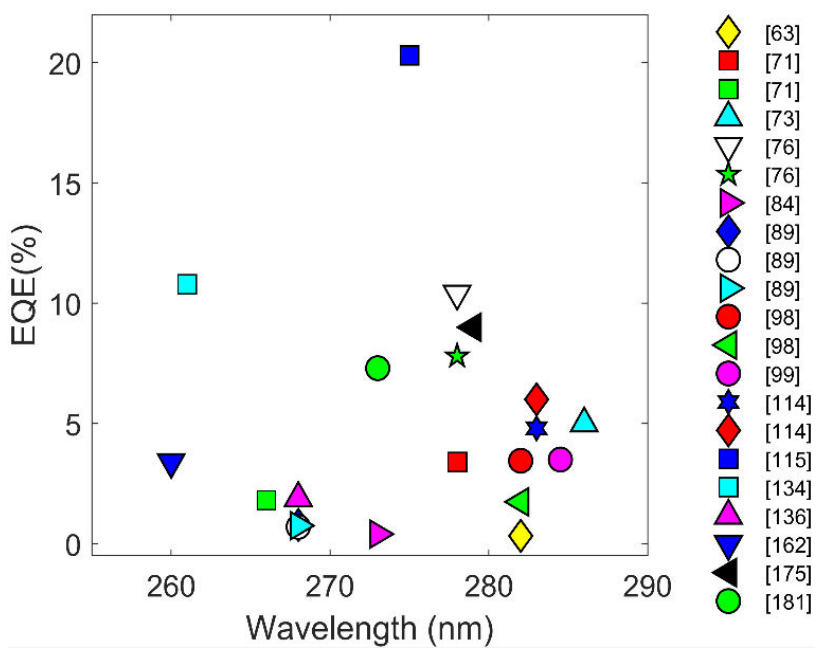

FIGURE 8. UVC LED EQE evolution.

electronic devices. For example, aiming for short-wavelength emission, thin wells with a high $\mathrm{Al}$ content are required, which increases the built-in electric field as stated above. To compensate, thin barriers could be used to mitigate such an increase.

On the other hand, the QCS effect is not present along the (100) direction of cubic-AlGaN. However, the emission wavelength in this phase is limited by the $\mathrm{Al}$ content in the barrier since $\mathrm{Al}_{x} \mathrm{Ga}_{1-x} \mathrm{~N}$ becomes an indirect band-gap material at $x=0.7$. Even so, it is possible to use cubic AlGaN material for UVC LEDs. For example, $260 \mathrm{~nm}$ emission can be obtained employing cubic $\mathrm{Al}_{0.7} \mathrm{Ga}_{0.3} \mathrm{~N}$ barriers and $\mathrm{Al}_{0.5} \mathrm{Ga}_{0.7} \mathrm{~N}$ wells with a thickness of $1.3 \mathrm{~nm}$. The variation of wavelength with well width is plotted in Fig. 8 for different $\mathrm{Al}$ content in cubic QWs. Additional advantages of employing the cubic phase are the easier p-type doping, higher mobility, and perpendicular $<011>$ cleavage directions [115]. To further enhance the efficiency, the QB must be designed in such a way that SRH recombination, tunneling leakage, and carrier overflow are mitigated [116].

On the other side, QDs are used to set the bandgap between desired values, with promising results from $232 \mathrm{~nm}$ to $270 \mathrm{~nm}$ [117]. This change in emission wavelength is made by controlling the thickness of GaN QD (Fig. 9) while keeping them between 1 and 4 monolayers (ML) for the GaN/AlN active region emitting at $225 \mathrm{~nm}$.

Even though hexagonal-phase LED development has advanced in the UVC range, it is possible to observe in Fig. 10 that some researchers still present EQE equal to $1 \%$ and $2 \%$ in wavelengths around $220 \mathrm{~nm}$ [118] and $280 \mathrm{~nm}$ [95], respectively. In order to increase these values, optimization of deposition techniques, materials, and the entire LED fabrication process has allowed witnessing increasingly constant reports with $\mathrm{EQE}$ around $10 \%$ [74, 119] and up to $20 \%$ after a full improvement with an emission peak centered at 275 $\mathrm{nm}$ [120]. It has been reported that when both LEE and IQE increase to $60 \%$, it was also necessary the addition a $50 \mathrm{~nm}$ $\mathrm{Al}_{0.65} \mathrm{Ga}_{0.35} \mathrm{~N}$ transparent contact layer doped with $\mathrm{Mg}$

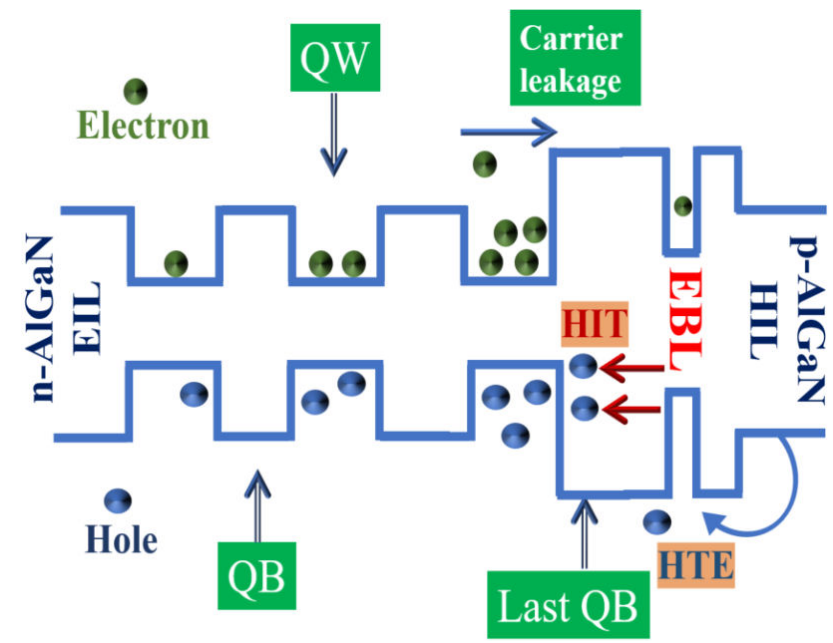

FIGURE 9. Schematic band diagram of MQW-based LED (without internal electric fields effect for simplicity).

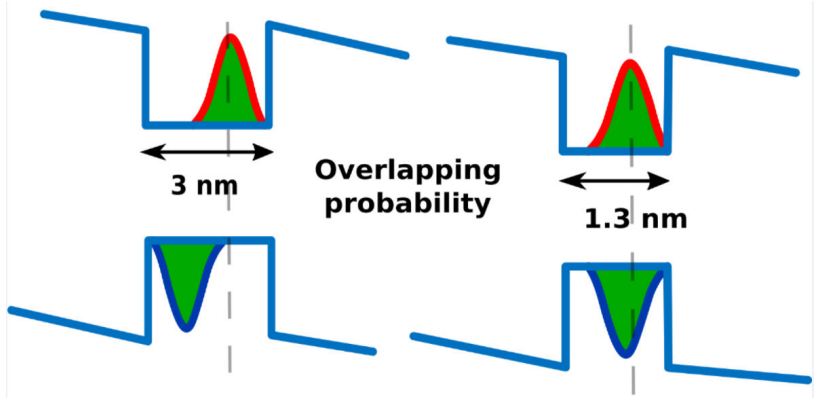

FIGURE 10. Effect of thickness of QW on the electron-hole overlapping probability.

(hole centration of $8 \times 10^{19} \mathrm{~cm}^{-3}$ ), a reflective Rhodium ( $\mathrm{Rh}$ ) electrode, a sapphire substrate, and encapsulation with resin. This IQE increment is due to the transparency enhancement of aluminum nitride templates [121]. Moreover, it has been reported that the use of uneven $\mathrm{AlGaN}$ QW with $\mathrm{Al}$ content over the $60 \%$ allow that IQE increases and, subsequently, the EQE [122].

\section{QW and QB roles in $\mathrm{UV}$ emission}

In III-Nitrides semiconductors, the asymmetrical behavior of electrons and holes, such as the difference in their effective mass and mobility, deteriorates the performance of devices [123]. Because of that, the concentration of carriers is not uniformly distributed inside of the QW region. Consequently, the radiative performance of the device is degraded especially at high current densities [124]. A number of the strategies are presented for the improvement of performance. In design, researchers are mainly focused on hole injection into the active region because of its high effective mass. Usually, in the conventional structure of UV-LEDs, a single layer for the electron blocking (EBL) is used, i.e., p-AlGaN, for better confinement of carriers inside of the active region.

The EBL provides a high barrier in the path of highenergy electrons, in this way, leakage of electrons from the 


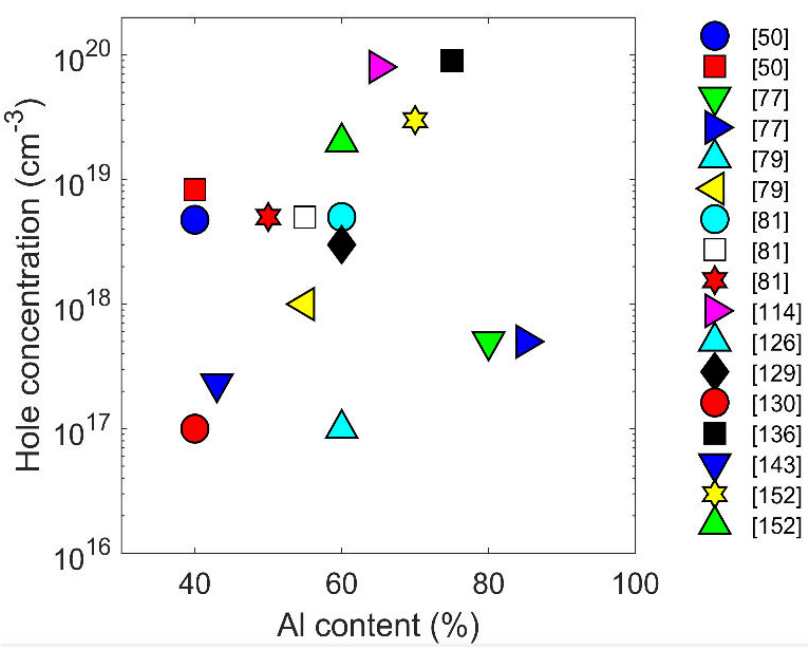

FIGURE 11. Hole concentration versus $\mathrm{Al}$ content in $\mathrm{AlGaN}$ alloys.

active region is reduced. Besides, EBL affects the hole injection efficiency into the active region due to barrier height. In this situation, most of the holes are injected by thermionic emission. The phenomena of hole injection by thermionic emission can be improved by interplaying two factors: hole concentration and valence band offset [125]. Moreover, the difference in the content of $\mathrm{Al}$ composition in the last $\mathrm{QB}$ and EBL leads to the formation of a polarization field. Various approaches have been reported in which holes are injected by both thermionic emission and intra-band tunneling. Khan et al. have reported the feasible solution for the reduction in $\mathrm{Mg}$-diffusion from $\mathrm{p}$-doped EBL to the active region and hole injection by intra-band tunneling [125]. They have reduced the thickness of the final QB (Al content same as in EBL) and introduced a valley in EBL with low Al content in comparison to EBL layers, simultaneously in the proposed device structure. For a better understanding, the schematic band diagram of the device structure is shown in Fig. 11. Consequently, the proposed structural strategy of the device is supported for the enhancement of intra-band tunneling (HIT) and hole by thermionic emission (HTE) because of the decrease in valence band offset, meanwhile the issue of uneven distribution of carriers inside the active region is also reduced.

Regarding the thickness of QWs and QB, several researchers have reported that thicker QW and QB leads to the uneven distribution of carriers inside the active region which degrades the radiative performance $[126,127]$. Due to thicker QB, the hole tunneling inside of the active region is reduced. Khan et al. have studied in detail the effect of the last QB thickness by introducing the last QB with different thicknesses $(4,6,8$, and $10 \mathrm{~nm})$. They concluded that the device structure with a thickness of $6 \mathrm{~nm}$ exhibited the best performance among all of them. Since for $4 \mathrm{~nm} \mathrm{QB}$, the chance of $\mathrm{Mg}$-diffusion towards the active region is also increased. Therefore, for the compensation of both, hole tunneling and Mg-diffusion, the thickness of $6 \mathrm{~nm}$ QB is reported to be the best.

\section{EBL and p-type layers}

Despite the increase in the IQE by reducing the dislocations, enhancing the active region and contact layers, electron leakage due to high operating currents and the associated heating affect the LED, causing an efficiency droop, decreasing the EQE to values lower than 5\% [128]. While p-type Al$\mathrm{GaN}$ layers with high $\mathrm{Al}$ content used as hole injection layer (which commonly goes under p-type contact layer shown in Fig. 3) have low hole concentration (lower than $1 \times 10^{17}$ $\mathrm{cm}^{-3}$ ) and will provoke parasitic emissions [129]. Enhancing the radiative recombination by increasing the carrier confinement in UVC LED also requires leakage electron blocking.

For performing the EBL, it is necessary high $\mathrm{Al}$ content to avoid that the electrons absorb energy without emitting the expected UVC radiation. However, high Al content drawbacks in design. To solve this, some approaches have been studied such as p-type electron blocking layer (p-EBL), pEBL superlattices, and graded superlattices [128-132]. By applying an EBL with an SL structure, the effective barrier height at the valence band is decreased. On the other hand, the conduction band is increased, mitigating the electron leakage while the hole injection augments [75]. It has been demonstrated that the inclusion of p-EBL superlattices to the LED structure allows achieving high EQE compared to similar LEDs without EBL 131 and even increasing around $90 \%$ more EQE by reducing the efficiency droop to $4 \%$ in LEDs emitting around $270 \mathrm{~nm}$ [128].

Another approach to improve LED performance is to substitute the conventional EBL with graded EBL, step graded and step graded superlattices, which have presented IQE up to $37.4 \%$ and $15 \%$ less efficiency droop with an emission peak at $224.76 \mathrm{~nm}$ [78]. Besides, the implementation of a layer that allows blocking the holes just like the EBL blocks the leaking electrons have been recently investigated. This Hole Blocking Layer (HBL) implementation has been demonstrated to enhance both the light-output power (24.2 $\mathrm{mW}$ to $56.1 \mathrm{~mW}$ ) and IQE (from $32 \%$ up to $72 \%$ ) [76]. In other words, by growing HBL between the active region and n-type layer, the radiative recombination augments, and the hole leakage is prevented $[76,77]$.

As mentioned above, EQE is an essential parameter to measure the efficiency of LED devices. Thus, different approaches to enhance this value are explored above. Another critical path is to replace the typically used $\mathrm{p}-\mathrm{GaN}$ with $\mathrm{p}$ $\mathrm{AlGaN}$ as a contact layer to reduce the UV absorption. However, this is a complicated task because of the high activation energy necessary to implant $\mathrm{Mg} 120$ from $160 \mathrm{meV}$ [133] to $500 \mathrm{meV}$ [134], even up to $630 \mathrm{meV}$ [135]. Despite this, it has been demonstrated that improving the transparency of contact layers enables the LED to increase EQE at least $5 \%$ by changing $\mathrm{Ni} / \mathrm{Au}$ electrodes for $\mathrm{Ni} / \mathrm{Al}$ electrodes [71, 136]. Also, EQE can be improved 3\% more through rising hole concentration in $\mathrm{p}-\mathrm{AlGaN}$, despite the drawbacks that 

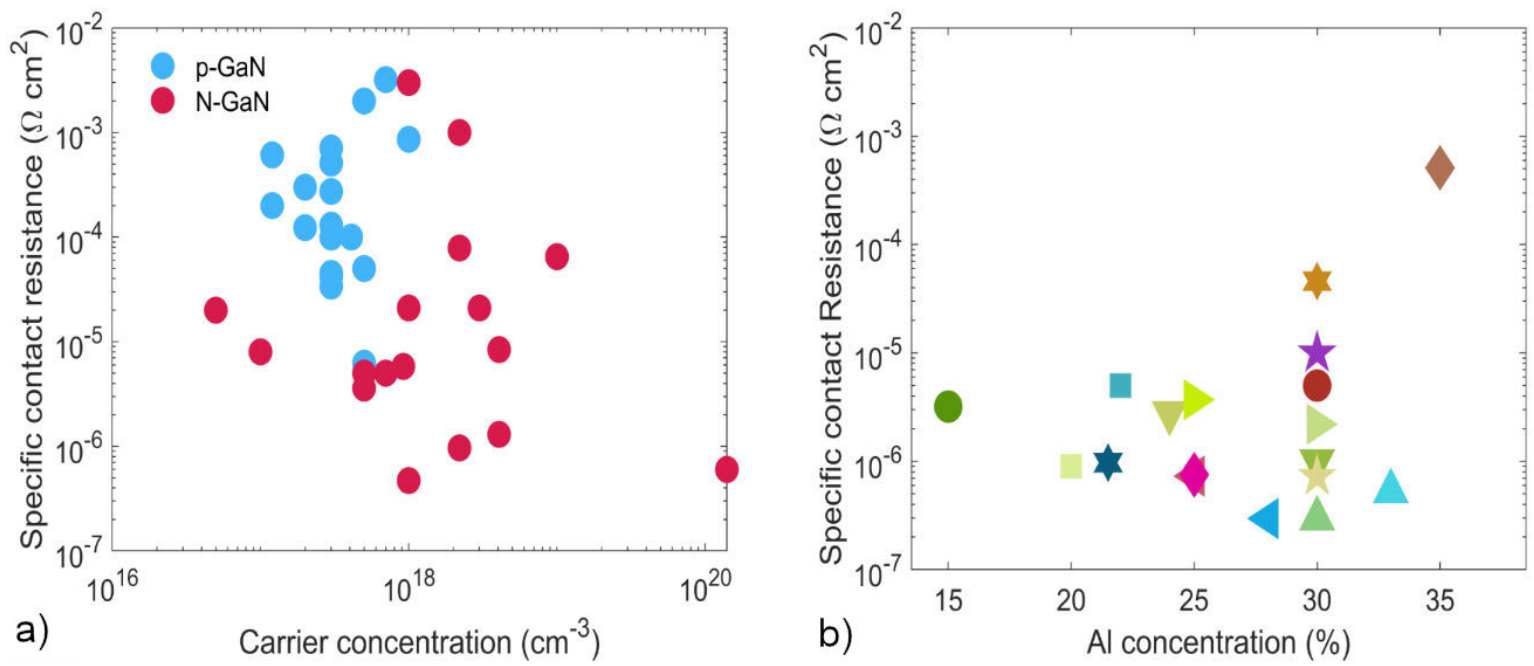

FIGURE 12. Specific contact resistance performance based on Greco et al[161], a) Differences between p-GaN against n-GaN heterostructure as the contact layer b) Al concentration vs Specific contact resistance in AlGaN/GaN heterostructures.

p-doping AlGaN:Mg involve, finally superseding GaN contact layer [71].

Until this point, the mechanism for enhancing efficiency in UVC LEDs have been discussed, considering the results depending on what previous works reported. However, several considerations must be done such as p-doping drawbacks when high $\mathrm{Al}$ content is used in AlGaN alloys, how this high $\mathrm{Al}$ content benefits the material transparency and what is needed to decrease the specific resistance of materials and allow a more efficient carrier injection to the active region $[117,137,138]$.

Whereas $\mathrm{p}-\mathrm{GaN}$ ohmic contact growth is studied to improve the efficiency [139], the use of AlGaN in the p-type doping and growth of ohmic contacts remains in search to become more functional. Although 7\% more EQE at $276 \mathrm{~nm}$ emission peak has been reported by the implementation of a $\mathrm{p}-\mathrm{AlGaN}$ transparent contact layer is implemented, when the wavelength is shortened to around $260 \mathrm{~nm}$, the EQE droops to $2.3 \%-3.3 \%[136,138]$. For the p-contact layer increases the transparency, it is necessary to substitute the typical GaN contact layer with $\mathrm{AlGaN}$ with high $\mathrm{Al}$ content; however, the more $\mathrm{Al}$ content is in the alloy, the greater activation energy for p-type doping is needed [140].

\section{P-type doping and ohmic contacts}

Since growth and doping required both accuracy and fine control of fluxes, epitaxy is used as the main deposition technique for LED fabrication. In this way, there are three epitaxial techniques more used such as molecular beam epitaxy (MBE), migration enhanced epitaxy (MEE), and metalorganic chemical vapor deposition (MOCVD) [141-144]. Besides, the development of combined methods based on MBE, MEE, and MOCVD, such as metal-source flow-rate modulation epitaxy, are of interest as long as Nitrogen flux remains constant reducing the activation energy of accep- tors and smooth surfaces could be produced [145]. On the other hand, besides the deposition technique used for growing, in order to obtain high p-type doping levels, it is necessary to consider that while cubic phase $\mathrm{p}-\mathrm{GaN}$ has demonstrated recently to reach an $\mathrm{Mg}$ concentration of $10^{20} \mathrm{~cm}^{-3}$ for III-V semiconductors, it must be considered that this value is approaching the solubility solution limit which could cause a strong drop in hole concentration because of selfcompensation that involves $M g-V_{N}$ complexes [146]. However, it is remarkable to note that hole concentration over $10^{19} \mathrm{~cm}^{-3}$ with a mobility of $3 \mathrm{~cm}^{2} / \mathrm{Vs}$ point to $\mathrm{c}-\mathrm{GaN}$ is a suitable alternative to obtain high free hole concentration for cubic-structures-based LEDs [146]. In addition, considering the current state of art emphasizes the use of hexagonal phase for UVC LED development, the doping of AlGaN is more complicated than GaN doping (p-type more restricted than n-type), because of the high activation energy of $\mathrm{Mg}$ around $630 \mathrm{meV}$ (for AlN:Mg) [135, 143], and the maximum solubility of dopants in III-nitrides semiconductors [105]. Thus, bulk structures, superlattices, grading the $\mathrm{Al}$ content, even $\delta$ doping techniques have been studied to increase the hole concentration without affecting other properties [134, 147, 148], and SL has been demonstrated to diminish the activation energy for doping improvement [149].

Although the drawbacks above, Fig. 12 suggest that the free hole concentration range of $10^{17}-10^{19} \mathrm{~cm}^{-3}$ is a reasonable interval to consider when it comes to the hole concentration for $\mathrm{Al}$ content between $50 \%$ to $80 \%$. Besides, it is important to mention that the material resistivity and transparency of $\mathrm{AlGaN}$ layers are augmented as long as $\mathrm{Al}$ content increases since the $\mathrm{Al}$ is added to the $\mathrm{GaN}$ the electronic mobility is diminished which results in a resistivity increase despite a high hole concentration (over $10^{19} \mathrm{~cm}^{-3}$ ) [150].

All growth and doping improvements have allowed that bulk structures are not the only option for UVC LED elaboration. For example, the accuracy provided by epitaxy makes 
it possible to create nanostructures and thin superlattices to set the energy band between the UVC range. The use of $\mathrm{Al}_{x} \mathrm{Ga}_{1-x} \mathrm{~N} / \mathrm{Al}_{y} \mathrm{Ga}_{1-y} \mathrm{~N}$ superlattices makes the resistivity less independent on temperature, increasing the free hole concentration even under low temperatures, reaching up a hole concentration of about $3.5 \times 10^{18} \mathrm{~cm}^{-3}$ without sacrificing resistivity based on $\mathrm{Al}_{0.63} \mathrm{Ga}_{0.37} \mathrm{~N} / \mathrm{Al}_{0.51} \mathrm{Ga}_{0.49} \mathrm{~N}$ superlattice structures [151]. Moreover, other works suggest the use of a quaternary alloy of AlInGaN to replace the p-AlGaN intermediate layer (between the active region and ohmic contact) to reduce the IQE droop by enhancing the hole injection to the active region [152].

A different approach, to upgrade the p-type doping results, is to gradually add Al to GaN and carry out the doping process to increase the LOP and reduce the forward bias polarization. At the same time, the disadvantages of the electron blocking layer are avoided $[153,154]$. When the Al content in a p-contact layer is graded, both the electron and hole concentration increase in the active region mainly due to the low effective potential height for holes which improves the hole injection to the active region [155]. Besides, the non-radiative recombination associated with holes and leaked electrons is reduced by the high effective electron barrier height, demonstrating the advantage of the graded active region over bulk layers $[155,156]$. Another structure with more efficient performance is the superlattice. In this structure the hole mobility is increased by a factor of five compared to a bulk structure, reducing the resistivity using polarization effects $[157,158]$.

As mentioned above, one more doping strategy is based on dopant distribution which is described by Dirac's $\delta$ function, the $\delta$-doping [159]. This has proven to be an effective method to improve the conduction of AlGaN p-type layers with high $\mathrm{Al}$ content by the self-compensation reduction whereas hole concentration increases 50 . This enhancement is due to the use of In as a surfactant, which allows that incorporation of $\mathrm{Mg}$ increases, diminishing the activation energy of the acceptors whereas p-type conductivity augments [50]. Furthermore, it is remarkable to mention that, in n-type, $\delta$-doping has presented results even more promising than graded or superlattices [160].

Ohmic contacts are the interface between electronic circuitry and the light-emitting device. Thus, the contact resistance is closely related to power losses of the entire system [161]. To achieve an efficient UVC LED, this should have low resistances in its contact layers. The n-type contact gives a massive component of resistance to the device when high $\mathrm{Al}$ content is used, thus, by using $\mathrm{Si}$ as n-AlGaN dopant the specific resistance of the material is reduced whereas the electron concentrations tends to $10^{19} \mathrm{~cm}^{-3}$ [162] The stateof-the-art presents free electron concentrations around $10^{18}$ $\mathrm{cm}^{-3}$ under $60 \%$ of $\mathrm{Al}$, this value, parallelly have reported contact resistance over $10^{-6} \Omega \mathrm{cm}^{2}$ by using $\mathrm{V} / \mathrm{Al} / \mathrm{Ni} / \mathrm{Au}$ electrodes [163]. Nevertheless, while Si doping levels rise, $\mathrm{n}$-AlGaN layers start to behave as an insulator due to the selfcompensation [164].
As Fig. 13a) shows, the p-type ohmic contact deposition is more complicated than the n-type because of the $\mathrm{p}$ doping drawbacks mentioned in the previous section. Commonly, p-GaN is used for UV LED despite its opacity resulting in low extraction of the light. However, it is also reported that $\mathrm{p}$-AlGaN contact layers have been shown to increase LEE [165, 166]. Moreover, superlattices based on $\mathrm{Al}_{x} \mathrm{Ga}_{1-x} \mathrm{~N} / \mathrm{Al}_{y} \mathrm{Ga}_{1-y} \mathrm{~N}$ or $\mathrm{Al}_{x} \mathrm{Ga}_{1-x} \mathrm{~N} / \mathrm{GaN}$ are used instead of the typical GaN p-type contact layer due to the tunability of the energy gap by changing the $\mathrm{Al}$ content and the lower resistance [167-170]. The specific contact resistance varies between $10^{-4} \Omega \mathrm{cm}^{2}$ and $10^{-7} \Omega \mathrm{cm}^{2}$, depending on the $\mathrm{Al}$ content in $\mathrm{AlGaN} / \mathrm{GaN}$, as shown in Fig. 13b) even without doping the $\mathrm{AlGaN} / \mathrm{GaN}$ heterostructure and considering the low concentration of $\mathrm{Al}$, the specific contact resistance remains lower than $\mathrm{p}-\mathrm{GaN}$ ones. Despite AlGaN/GaN heterostructures contact resistance is lower than $\mathrm{GaN}$ resistance as shown in Fig. 13a), it is useful to growth a thin p-GaN layer to guarantee Ohmic contact deposition without depending on negative effects caused by the addition of $\mathrm{Al}$ [166], a thin p-GaN layer $(<10 \mathrm{~nm})$ over the superlattice has also been used as a low resistive ohmic contact [171]. On the other side, to improve the EQE despite a low hole concentration in $\mathrm{p}-\mathrm{A}_{l 0.6} \mathrm{Ga}_{0.4} \mathrm{~N}$ doped with $\mathrm{Mg}$, the use of High $\mathrm{Re}$ flective Photonic Crystals (HR-PhC), over the p-contact layer have been proposed to facilitate the tunneling effect which enhances the LEE [70] even though hole concentration drops down to $10^{3} \mathrm{~cm}^{-3}$ [119].

Once the contact layer has been grown and the n-contact layer is exposed by wet or dry etching techniques [172-174], ohmic contacts that provide the correct electron injection to the active region must be deposited. The most used metals are Nickel/Gold (Ni/Au) [139, 163, 170, 175], this is because the $\mathrm{Ni}$ work function is greater than the p-contact layer work function, avoiding that a Schottky barrier is generated on the p-type material $[176,177]$ and also it has been reported that as the $\mathrm{Ni}$ thickness decreases to around $0.8 \mathrm{~nm}$, the reflectivity augments [178]. However, elements such as Vanadium, Platinum, and Rhodium (V, Pt, Rh), are used to diminish the specific contact layer [163, 164, 179, 180], by substituting Au with Silver (Ag) the specific contact layer is comparable with those that use $\mathrm{Au}$ [171], even more useful due to the increase of reflectivity reaching contact resistance up to $3.5 \times 10^{-5}$ $\Omega \mathrm{cm}^{2}[181]$.

\section{Conclusions}

In summary, to increase UVC-LEDs' efficiency for germicidal applications, several considerations must be performed. First, it is essential to deposit a buffer layer that absorbs strains to reduce the propagation of structural defects to the next layers [102]. Most of the works reported in the stateof-the-art point out the hexagonal crystal structure for UVC devices mainly due to the cubic phase direct-indirect bandgap cross point around $70 \%$ of $\mathrm{Al}$ in $\mathrm{AlGaN}$ alloys 56. However, the development of c-AlGaN-based UVC LED is feasible if 
the quantum confinement is increased despite the reduced $\mathrm{Al}$ content available before exceeding this cross point by employing thin nanostructures such as $1.3 \mathrm{~nm}$-thick QW with $\mathrm{Al}$ content around $50 \%$ and $70 \%$ for $\mathrm{QW}$ and $\mathrm{QB}$, respectively, in the active region emitting at $260 \mathrm{~nm}$, as suggested by figure 8 . It must be considered that while the wavelength increases from $260 \mathrm{~nm}$, greater doses are needed to reduce the virus load 26. Furthermore, the $\mathrm{Al}_{x} \mathrm{Ga}_{1-x} \mathrm{~N}$ p-type doping is limited by the $\mathrm{Al}$ molar fraction $(x)$ due to the activation energy increases as the $\mathrm{Al}$ content increases reaching to $\sim 630 \mathrm{meV}$ for h-AlN $(x=1)[135,143]$; nevertheless, different approaches to diminish the high $\mathrm{Al}$ content effects are implemented $[151-153,155,156]$ allowing to conclude that the superlattices structures $[151,152,182]$ and Al content graded p-regions $[153,155]$ are the most promising approaches for the p-type doping by decreasing activation energy [149]. The typical p-GaN contact layer must be substituted with a more transparent highly doped p-AlGaN hole transport layer to ensure low contact resistance by selecting the appropriate electrodes [164-171]. It is remarkable mentioning that the UVC-LED EQE record is $20.3 \%$ emitting a UVC radiation centered on $275 \mathrm{~nm}$, by employing a sapphire patterned substrate to reduce threading dislocations, substituting $\mathrm{p}-\mathrm{GaN}$ for $\mathrm{p}-\mathrm{AlGaN}$ to reduce the absorption in the contact layer, the use of $\mathrm{Rh}$ mirror electrode and HR-PhC to increase reflectivity and resin coating. However, we suggested in addition to previously discussed efforts for an EQE of $20 \%$, the use of superlattices in the EBL and p-cladding layer could improve the $\mathrm{EQE}$ due to a reduced hole leakage and increased hole injection, respectively. Moreover, the advantages of the cubic phase over the most studied hexagonal one, such as low Mg activation energy due to crystal symmetry [146], the absence of spontaneous and piezoelectric fields in the active region (as discussed in Fig. 7), and the possibility to emit UVC-radiation supported by our QW calculations without exceeding direct-to-indirect band gap cross point limit with an expected emission at $260 \mathrm{~nm}$ are strong arguments to consider the c-AlGaN UVC-LED as an emergent candidate to overcome the current EQE.

\section{Acknowledgments}

The authors are grateful to the Tecnológico Nacional de México and Instituto Politécnico Nacional for their entire support to develop healthcare technology in times of crisis. Moreover, the authors want to thank Vanessa Cardona for her biology discussions.

Funding: This work was supported by the Consejo Nacional de Ciencia y Tecnología (CONACYT) in Mexico through its National Scholarship Program.

\section{Declaration of interests}

The authors declare that they have no known competing financial interests or personal relationships that could have appeared to influence the work reported in this paper.
1. World Health Organization, WHO Coronavirus Dis. Dashboard (2020), https://covid19.who.int/table

2. H. Jiang, Y. Zhou, and W. Tang, Maintaining HIV care during the COVID-19 pandemic, Lancet HIV 7 (2020) (5), https: //doi.org/10.1016/S2352-3018(20)30105-3

3. N. E. Sharpless, COVID-19 and cancer, Science (2020) 368 (2020) 1290, https://doi.org/10.1126/science. abd3377

4. A. Kutikov et al., A War on Two Fronts: Cancer Care in the Time of COVID-19, Ann. Intern. Med. 172 (2020) 756, https://doi.org/10.7326/M20-1133

5. J. van de Haar et al., Caring for patients with cancer in the COVID-19 era, Nat. Med. 26 (2020), https://doi.org/ $10.1038 / \mathrm{s} 41591-020-0874-8$

6. Wold Health Organization, The Impact of the COVID-19 Pandemic on Noncommunicable Disease Resources and Services: Results of a Rapid Assessment, Geneva, Switzerland (2020), https://www.who.int/publications/ i/item/9789240010291

7. B. Marroquón, V. Vine, and R. Morgan, Mental health during the COVID-19 pandemic: Effects of stay-at-home policies, social distancing behavior, and social resources, Psychiatry Res. 293 (2020), https://doi.org/10.1016/j. psychres.2020.113419
8. X. Xie, et al., Mental Health Status Among Children in Home Confinement During the Coronavirus Disease 2019 Outbreak in Hubei Province, China, JAMA Pediatr. 174 (2020) 898, https://dx.doi.org/10.1001/ jamapediatrics.2020.1619.

9. Institute for Economics and Peace, Global Peace Index 2020: Measuring Peace in a Complex World, Sidney (2020).

10. World Bank, Poverty and Shared Prosperity 2020: Reversals of Fortune, World Bank, The World Bank, Washington, DC (2020).

11. World Health Organization, Advice on the use of masks in the context of COVID-19: interim guidance, World Heal. Organ. (2020), https://apps.who.int/iris/handle/ $10665 / 331693$

12. S. Ugalmugle, and R. Swain, Protective Face Masks Market, Glob. Mark. Insights (2020).

13. S. Mehta et al., Community-Based Production of Quality Face Masks for Personal Protection During the COVID-19 Pandemic, Am. J. Med. Qual. 36 (2020) 17, https://doi. org/10.1177/1062860620956726

14. T. A. Aragaw, Surgical face masks as a potential source for microplastic pollution in the COVID-19 scenario. Mar. Pollut. Bull. 159 (2020) 111517, https://doi.org/10.1016/ j.marpolbul.2020.111517 
15. C. E. Rodriguez-Martinez, M. P. Sossa-Briceño, and J. A. Cortés, Decontamination and reuse of N95 filtering facemask respirators: A systematic review of the literature, Am. J. Infect. Control 48 (2020) 1520, https://doi.org/10.1016/ j.ajic.2020.07.004

16. H. $\mathrm{Vu}$ et al., Design and Evaluation of Uniform LED Illumination Based on Double Linear Fresnel Lenses, Appl. Sci. 10 (2020) 3257, https://doi.org/10.3390/ app10093257

17. E. Espid, and F. Taghipour, UV-LED Photo-activated Chemical Gas Sensors: A Review, Crit. Rev. Solid State Mater. Sci. 42 (2017) 416, https://doi.org/10.1080/10408436. 2016.1226161

18. P. O. Nyangaresi et al., Comparison of the performance of pulsed and continuous UVC-LED irradiation in the inactivation of bacteria, Water Res. 157 (2019) 218, https : / / doi. org/10.1016/j.watres.2019.03.080

19. C. S. Heilingloh et al., Susceptibility of SARS-CoV-2 to UV irradiation. Am. J. Infect. Control 48 (2020) 1273, https: //doi.org/10.1016/j.ajic.2020.07.031

20. M. Eickmann et al., Inactivation of Ebola virus and Middle East respiratory syndrome coronavirus in platelet concentrates and plasma by ultraviolet $\mathrm{C}$ light and methylene blue plus visible light, respectively, Transfusion 58 (2018) 2202, https : / / doi.org/https://doi.org/10.1111/trf.14652

21. J.-L. Sagripanti, and C. D. Lytle, Sensitivity to ultraviolet radiation of Lassa, vaccinia, and Ebola viruses dried on surfaces, Arch. Virol. 156 (2011) 489, https : / / doi .org/10. 1007/s00705-010-0847-1.

22. C. A. Bolton J R and Cotton, The Ultraviolet Disinfection Handbook, 1st ed. (American Water Works Association, 2008).

23. A. Green, V. Popović, K. Warriner, and T. Koutchma, The efficacy of UVC LEDs and low pressure mercury lamps for the reduction of Escherichia coli O157:H7 and Listeria monocytogenes on produce, Innov. Food Sci. Emerg. Technol. 64 (2020) 102410, https://doi.org/10.1016/j. ifset.2020.102410

24. D. kyun Kim, and D. H. Kang, Effect of surface characteristics on the bactericidal efficacy of UVC LEDs, Food Control 108 (2020) 106869, https://doi.org/10.1016/ j.foodcont.2019.106869.

25. J. Chen, S. Loeb, and J. H. Kim, LED revolution: Fundamentals and prospects for UV disinfection applications, Environ. Sci. Water Res. Technol. 3 (2017) 188, https://doi .org/ $10.1039 / \mathrm{c} 6 \mathrm{ew} 00241 \mathrm{~b}$

26. Y. Gerchman, H. Mamane, N. Friedman, and M. Mandelboim, UV-LED disinfection of Coronavirus: Wavelength effect, J. Photochem. Photobiol. B Biol. 212 (2020) 112044, https://doi.org/https://doi.org/10. 1016/j.jphotobiol.2020.112044

27. A. Ringangaonkar, and S. N. Kulkarni, A Comparative Study on UVC Light Devices To Inactivate Viruses, Int. Res. J. Mod. Eng. Technol. Sci. 2 (2020).

28. M. Raeiszadeh, and B. Adeli, A Critical Review on Ultraviolet Disinfection Systems against COVID-19 Outbreak: Applicability, Validation, and Safety Considerations, ACS Photonics 7 (2020) 2941, https://doi.org/10.1021/ acsphotonics.0c01245
29. A. J. Prussin, E. B. Garcia, and L. C. Marr, Total concentrations of virus and bacteria in indoor and outdoor air, Environ. Sci. Technol. Lett. 2 (2015) 84, https : / / doi.org/10.1021/ acs.estlett.5b00050.

30. A. Barlev, and D. Sen, DNA's Encounter with Ultraviolet Light: An Instinct for Self-Preservation? Acc. Chem. Res. 51 (2018) 526, https://doi.org/10.1021/acs. accounts.7b00582

31. R. Verbeke, I. Lentacker, S. C. De Smedt, and H. Dewitte, Three decades of messenger RNA vaccine development, Nano Today 28 (2019) 100776, https://doi.org/https:// doi.org/10.1016/j.nantod.2019.100766

32. T. Schlake, A. Thess, M. Fotin-Mleczek, and K.-J. Kallen, Developing mRNA-vaccine technologies, RNA Biol. 9 (2012) 1319, https://doi.org/10.4161/rna.22269.

33. J. Cohen, Vaccine designers take first shots at COVID19, Science 368 (2020), https://doi.org/10.1126/ science.368.6486.14

34. P. Midoux, and C. Pichon, Lipid-based mRNA vaccine delivery systems, Expert Rev. Vaccines 14 (2015) 221, https: //doi.org/10.1586/14760584.2015.986104

35. F. Krammer, SARS-CoV-2 vaccines in development. Nature 586 (2020) 516, https://doi.org/10.1038/ s41586-020-2798-3

36. G. Chauhan et al., Nanotechnology for COVID-19: Therapeutics and Vaccine Research, ACS Nano 14 (2020) 7760, https : //doi.org/10.1021/acsnano.0c04006

37. S. Duffy, Why are RNA virus mutation rates so damn high?, PLOS Biol. 16 (2018) e3000003, https : // doi.org/10. $1371 /$ journal.pbio.3000003

38. Q. Li et al., The Impact of Mutations in SARS-CoV2 Spike on Viral Infectivity and Antigenicity, Cell 182 (2020) 1284, https://doi.org/https://doi.org/ $10.1016 / j . c e l 1.2020 .07 .012$

39. J. Hu et al., D614G mutation of SARS-CoV-2 spike protein enhances viral infectivity, bioRxiv (2020) 161323, https: //doi.org/10.1101/2020.06.20.161323

40. W. Kowalski, Ultraviolet Germical Irradiation, Springer-Verlag Berlin Heidelberg, New York (2009), https://doi.org/ 10.1007/978-3-642-01999-9

41. M. Heßling, K. Hönes, P. Vatter, and C. Lingenfelder, Ultraviolet irradiation doses for coronavirus inactivation - review and analysis of coronavirus photoinactivation studies, GMS Hyg. Infect. Control 15 (2020), https://dx.doi.org/ $10.3205 / \mathrm{dgkh} 000343$.

42. Y. Cao, W. Chen, M. Li, B. Xu, J. Fan, and G. Zhang, Simulation Based Design of Deep Ultraviolet LED Array Module Used in Virus Disinfection., 2020 21st Int. Conf. Electron. Packag. Technol. (2020) 1, https://doi.org/10.1109/ icept50128.2020.9202924

43. M. Umar, F. Roddick, and L. Fan, Comparison of UVC Lamp and UVC-light Emitting Diodes for Treating Municipal Wastewater Reverse Osmosis Concentrate,International Conference on Biological, Civil and Environmental Engineering (2014) 18, https://doi.org/10.15242/iicbe. c0314076 
44. S. S. Nunayon, H. Zhang, and A. C. K. Lai, Comparison of disinfection performance of UVC-LED and conventional upper-room UVGI systems. Indoor Air, 30(2020)180https : //doi.org/10.1111/ina.12619

45. M. Lindblad, E. Tano, C. Lindahl, and F. Huss, UltravioletC decontamination of a hospital room: Amount of UV light needed, Burns 46 (2020) 842, https://doi.org/10. $1016 / j$.burns.2019.10.004

46. A. E. Torres, A. B. Lyons, S. Narla, I. Kohli, I. Kohli, A. ParksMiller, D. Ozog, I. H. Hamzavi, and H. W. Lim, Ultraviolet$\mathrm{C}$ and other methods of decontamination of filtering facepiece N-95 respirators during the COVID-19 pandemic, Photochem. Photobiol. Sci. 19 (2020) 746, https : / / doi . org/ $10.1039 / \mathrm{d} 0 \mathrm{pp} 00131 \mathrm{~g}$

47. L. Liao, W. Xiao, M. Zhao, X. Yu, H. Wang, Q. Wang, S. Chu, and Y. Cui, Can N95 Respirators Be Reused after Disinfection? How Many Times? ACS Nano 14 (2020) 6348, https://doi.org/10.1021/acsnano.0c03597

48. Y. Xiao, X. N. Chu, M. He, X. C. Liu, and J. Y. Hu, Impact of UVA pre-radiation on UVC disinfection performance: Inactivation, repair and mechanism study, Water Res. 141 (2018) 279, https://doi.org/10.1016/j.watres.2018. 05.021 .

49. H. P. Maruska, and J. J. Tietjen, The preparation and properties of vapor-deposited single-crystal-line GaN, Appl. Phys. Lett. 15 (1969) 327, https://doi.org/10.1063/1.1652845

50. Y. Chen, H. Wu, E. Han, G. Yue, Z. Chen, Z. Wu, G. Wang, and $\mathrm{H}$. Jiang, High hole concentration in p-type AlGaN by indiumsurfactant-assisted Mg-delta doping, Appl. Phys. Lett. 106 (2015) 8, https ://doi.org/10.1063/1.4919005

51. T. D. Moustakas, Ultraviolet optoelectronic devices based on AlGaN alloys grown by molecular beam epitaxy. MRS Commun. 6 (2016) 247, https://doi.org/10.1557/mrc. 2016.26

52. M. Kneissl, and J. Rass, III-Nitride Ultraviolet Emitters, (2016), https://doi.org/10.1007/ 978-3-319-24100-5_15.

53. J. Cho, J. H. Park, J. K. Kim, and E. F. Schubert, White lightemitting diodes: History, progress, and future, Laser Photonics Rev. 11 (2017) 1600147, https://doi.org/10.1002/ lpor.201600147

54. D. Feezell, and S. Nakamura, Invention, development, and status of the blue light-emitting diode, the enabler of solidstate lighting. Comptes Rendus Phys. 19 (2018) 113, https: //doi.org/10.1016/j.crhy.2017.12.001

55. J. Qiao, J. Zhao, Q. Liu, and Z. Xia, Recent advances in solid-state LED phosphors with thermally stable luminescence, J. Rare Earths 37 (2019) 565, https://doi.org/10. $1016 / j \cdot j r e .2018 .11 .001$

56. Y. C. Tsai, and C. Bayram, Structural and Electronic Properties of Hexagonal and Cubic Phase AlGaInN Alloys Investigated Using First Principles Calculations, Sci. Rep. 9 (2019) 1, https://doi.org/10.1038/ s41598-019-43113-w

57. H. Gao, H. Ye, Z. Yu, Y. Zhang, Y. Liu, and Y. Li, Point defects and composition in hexagonal group-III nitride monolayers: A first-principles calculation Superlattices Microstruct.
112 (2017) 136, https://doi.org/10.1016/j.spmi. 2017.09 .022

58. F. Li, L. Wang, G. Zhao, Y. Meng, H. Li, Y. Chen, S. Yang, P. Jin, and Z. Wang, The Residual Stress and Al Incorporation of AlGaN Epilayers by Metalorganic Chemical Vapor Deposition, J. Nanosci. Nanotechnol. 18 (2018) 7484, https: //doi.org/10.1166/jnn.2018.16055

59. I. Vurgaftman, J. R. Meyer, and L. R. Ram-Mohan, Band parameters for III-V compound semiconductors and their alloys, J. Appl. Phys. 89(2001)5815, https://doi.org/ $10.1063 / 1.1368156$

60. I. Vurgaftman, and J. R. Meyer, Band parameters for nitrogencontaining semiconductors, J. Appl. Phys. 94 (2003) 3675, https://doi.org/10.1063/1.1600519

61. L. Zhao et al., AlGaN-based ultraviolet light-emitting diodes on sputter-deposited AlN templates with epitaxial AlN/AlGaN superlattices, Superlattices Microstruct. 113(2018)713, https: //doi.org/10.1016/j.spmi.2017.12.003

62. Y. Hou, and Z. Guo, Enhancement of hole injection in deep ultraviolet light-emitting diodes using a serrated P-type layer, Opt. Commun. 433 (2019) 236, https://doi.org/https://doi.org/10.1016/ j.optcom.2018.10.024

63. Y. Kuo, J. Chang, F. Chen, Y. Shih, and H. Chang, Numerical Investigation on the Carrier Transport Characteristics of AlGaN Deep-UV Light-Emitting Diodes, IEEE J. Quantum Electron. 52(2016)1, https://doi.org/10.1109/ JQE.2016.2535252

64. H. Hirayama, Y. Tsukada, T. Maeda, and N. Kamata, Marked Enhancement in the Efficiency of Deep-Ultraviolet AlGaN Light-Emitting Diodes by Using a Multiquantum-Barrier Electron Blocking Layer. Appl. Phys. Express 3 (2010) 31002, https://doi.org/10.1143/apex.3.031002

65. T. Kolbe, J. Stellmach, F. Mehnke, M.-A. Rothe, V. Kueller, A. Knauer, S. Einfeldt, T. Wernicke, M. Weyers, and M. Kneissl, Efficient carrier-injection and electron-confinement in UV-B light-emitting diodes, Phys. status solidi 213 (2016) 210, https://doi.org/10.1002/pssa.201532479

66. M. Shatalov, W. Sun, R. Jain, A. Lunev, X. Hu, A. Dobrinsky, Y. Bilenko, J. Yang, G. A. Garrett, L. E. Rodak, M. Wraback, M. Shur, and R. Gaska, High power AlGaN ultraviolet light emitters,Semicond. Sci. Technol. 29 (2014) 84007, https: //doi.org/10.1088/0268-1242/29/8/084007.

67. J. Smalc-Koziorowska, J. Moneta, P. Chatzopoulou, I. G. Vasileiadis, C. Bazioti, O. Prytz, I. Belabbas, P. Komninou, and G. P. Dimitrakopulos, The heterogeneous nucleation of threading dislocations on partial dislocations in III-nitride epilayers, Sci. Rep. 10 (2020) 17371, https://doi.org/10.1038/ s41598-020-74030-y

68. X. Li et al., AlGaN-based MQWs grown on a thick relaxed AlGaN buffer on AlN templates emitting at $285 \mathrm{~nm}$, Opt. Mater. Express 5(2015)380, https://doi.org/10. $1364 / \mathrm{OME} .5 .000380$

69. M. Kim et al., AlGaN-Based Deep Ultraviolet Light-Emitting Diodes Fabricated on Patterned Sapphire Substrates. Appl. Phys. Express 4 (2011) 92102, https://doi.org/10. 1143 /apex.4.092102. 
70. W. Shin, A. Pandey, X. Liu, Y. Sun, and Z. Mi, Photonic crystal tunnel junction deep ultraviolet light emitting diodes with enhanced light extraction efficiency, Opt. Express 27 (2019) 38413, https://doi.org/10.1364/oe.380739.

71. N. Maeda, and H. Hirayama, Improvement of Ligth-Extraction Efficiency of Deep-UV LEDs using Transparent p-AlGaN Contact Layer, Conf. Lasers Electro-Optics Pacific Rim, Kyoto, Japan (2013), https://doi.org/10.1002/pssc. 201300278 .

72. B. K. SaifAddin, A. Almogbel, C. J. Zollner, H. Foronda, A. Alyamani, A. Albadri, M. Iza, S. Nakamura, S. P. DenBaars, and J. S. Speck, Fabrication technology for high lightextraction ultraviolet thin-film flip-chip (UV TFFC) LEDs grown on SiC, Semicond. Sci. Technol. 34 (2019) 35007, https://doi.org/10.1088/1361-6641/aaf58f

73. L. Anderson, W. Lee, W. Schaff, and J. C. Tang, US 10,516,076 B2 (2019).

74. M. Shatalov et al., AlGaN deep-ultraviolet light-emitting diodes with external quantum efficiency above 10\%, Appl. Phys. Express 5 (2012) 6, https://doi.org/10.1143/ APEX.5.082101

75. P. Sun et al., Advantages of AlGaN-based deep ultraviolet lightemitting diodes with a superlattice electron blocking layer, $\mathrm{Su}$ perlattices Microstruct. 85(2015)59, https://doi.org/ $10.1016 / j . s p m i .2015 .05 .010$

76. H. Shi et al., Performance improvements of AlGaN-based deep-ultraviolet light-emitting diodes with specifically designed irregular sawtooth hole and electron blocking layers, Opt. Commun. 441 (2019) 149, https://doi.org/10. $1016 /$ j.optcom.2019.02.054

77. L. Wang, W. He, T. Zheng, Z. Chen, and S. Zheng, Enhanced optical performance of AlGaN-based deep-ultraviolet light-emitting diode with m-shaped hole blocking layer and w-shaped electron blocking layer, Superlattices Microstruct. 133 (2019), 106188, https://doi.org/10.1016/j. spmi.2019.106188.

78. R. K. Mondal, V. Chatterjee, and S. Pal, Effect of step-graded superlattice electron blocking layer on performance of AlGaN based deep-UV light emitting diodes, Phys. E Low-dimensional Syst. Nanostructures 108(2019)233, https://doi.org/ $10.1016 / j \cdot$ physe.2018.11.022

79. J. Zhang, W. Tian, F. Wu, W. Yan, H. Xiong, J. Dai, Y. Fang, $\mathrm{Z}$. Wu, and C. Chen, The advantages of AlGaN-based UVLEDs inserted with a p-AlGaN layer between the EBL and active region, IEEE Photonics J. 5 (2013), 1600310, https: //doi.org/10.1109/JPHOT.2013.2278520

80. F. Scholz, Compound Semiconductors: Physics, Technology, and Device Concepts, Jenny Stanford Publishing (2017).

81. Y. Liao, C. Thomidis, C. K. Kao, and T. D. Moustakas, AlGaN based deep ultraviolet light emitting diodes with high internal quantum efficiency grown by molecular beam epitaxy, Appl. Phys. Lett. 98 (2011) 98, https://doi.org/10.1063/ 1.3559842 .

82. T. D. Moustakas, Y. Liao, C. Kao, C. Thomidis, A. Bhattacharyya, D. Bhattarai, and A. Moldawer, Deep UV-LEDs with high IQE based on AlGaN alloys with strong band structure potential fluctuations. Light. Diodes Mater. Devices,
Appl. Solid State Light. 8278 (2012) https://doi.org/ $10.1117 / 12.916213$

83. Y. Muramoto, M. Kimura, and S. Nouda, Development and future of ultraviolet light-emitting diodes: UV-LED will replace the UV lamp, Semicond. Sci. Technol. 29 (2014), https: //doi.org/10.1088/0268-1242/29/8/084004

84. A. S. Evseenkoy, S. A. Tarasov, A. Lamkin, A. V Solomonov, and I. Member, The Efficiency of UV LEDs Based on GaN / AIGaN Heterostructures., 2015 IEEE NW Russ. Young Res. Electr. Electron. Eng. Conf. (2015) 27, https : //doi.org/ 10.1109/EIConRusNW.2015.7102224

85. J. Rass, and N. L. Ploch, Nitride-Based UV-LEDs and Their Application, Opt. Photonik 11(2016)36, https://doi. org/10.1002/opph.201600020

86. S. Hagedorn et al., Improving AIN Crystal Quality and Strain Management on Nanopatterned Sapphire Substrates by HighTemperature Annealing for UVC Light-Emitting Diodes, Phys. Status Solidi Appl. Mater. Sci. 217 (2020) 1, https : //doi. org/10.1002/pssa.201900796

87. N. Susilo et al., AlGaN-based deep UV LEDs grown on sputtered and high temperature annealed AlN/sapphire, Appl. Phys. Lett. 112 (2018), https://doi.org/10.1063/1. 5010265

88. A. Ganguly, S. Chattopadhyay, K. H. Chen, and L. C. Chen, Production and storage of energy with one-dimensional semiconductor nanostructures, Crit. Rev. Solid State Mater. Sci. 39 (2014) 109, https://doi.org/10.1080/10408436. 2013.796909

89. E. F. de Almeida, F. de Brito Mota, C. M. C. de Castilho, A. Kakanakova-Georgieva, and G. K. Gueorguiev, Defects in hexagonal-AIN sheets by first-principles calculations, Eur. Phys. J. B 85 (2012) 48, https://doi.org/10.1140/ epjb/e2011-20538-6

90. A. Uedono, S. Ishibashi, N. Oshima, R. Suzuki, and M. Sumiya, Point Defect Characterization of Group-III Nitrides by Using Monoenergetic Positron Beams. ECS Trans. 61 (2014) 19, https://doi.org/10.1149/06105.0019ecst

91. M. Usman, S. Malik, and M. Munsif, AlGaN-based ultraviolet light-emitting diodes: challenges and opportunities, $\mathrm{Lu}$ minescence 36 (2021) 294, https://doi.org/https: //doi.org/10.1002/bio.3965

92. Y. Nagasawa, and A. Hirano, A Review of AlGaNBased Deep-Ultraviolet Light-Emitting Diodes on Sapphire, Appl. Sci. 8 (2018) 1264, https://doi.org/10.3390/ app8081264

93. B. K. Saifaddin et al., AlGaN Deep-Ultraviolet LightEmitting Diodes Grown on $\mathrm{SiC}$ Substrates, ACS Photonics 7 (2020) 554, https://doi.org/10.1021/ acsphotonics.9b00600

94. M. Usman, M. Munsif, U. Mushtaq, A.-R. Anwar, and N. Muhammad, Green gap in GaN-based light-emitting diodes: in perspective, Crit. Rev. Solid State Mater. Sci. (2020) 1, https: //doi.org/10.1080/10408436.2020.1819199

95. M. Kneissl et al., Advances in group III-nitride-based deep UV light-emitting diode technology, Semicond. Sci. Technol. 26 (2011), https://doi.org/10.1088/0268-1242/ 26/1/014036 
96. P. Dong et al., 282-nm AlGaN-based deep ultraviolet lightemitting diodes with improved performance on nano-patterned sapphire substrates, Appl. Phys. Lett. 102 (2013) 1, https: //doi.org/10.1063/1.4812237

97. J. Yan et al., AlGaN-based deep-ultraviolet light-emitting diodes grown on High-quality AlN template using MOVPE, J. Cryst. Growth 414 (2015) 254, https: / / doi.org/10. $1016 / j \cdot j c r y s g r o .2014 .10 .015$

98. J. Zhao et al., Heteroepitaxial growth of high-quality and crackfree AlN film on sapphire substrate with nanometer-scale-thick AlN nucleation layer for AlGaN-based deep ultraviolet lightemitting diodes, Nanomaterials 9 (2019), https://doi. org/10.3390/nano9111634

99. H.-M. Wang et al., AlN/AlGaN superlattices as dislocation filter for low-threading-dislocation thick AlGaN layers on sapphire, Appl. Phys. Lett. 81(2002)604, https://doi .org/ $10.1063 / 1.1494858$

100. J. Kim, J. Pyeon, M. Jeon, and O. Nam, Growth and characterization of high quality AlN using combined structure of low temperature buffer and superlattices for applications in the deep ultraviolet, Jpn. J. Appl. Phys. 54(2015)81001, https: //doi.org/10.7567/jjap.54.081001

101. M. Kaneko, S. Ueta, M. Horita, T. Kimoto, and J. Suda, Deep-ultraviolet light emission from $4 \mathrm{H}-\mathrm{AlN} / 4 \mathrm{H}-\mathrm{GaN}$ shortperiod superlattice grown on $4 \mathrm{H}-\mathrm{SiC}\left(112 \hat{\mathrm{A}}^{-0}\right)$, Appl. Phys. Lett. 112 (2018)12106, https: / /doi.org/10.1063/1. 5006435

102. Y. Huang et al., Crack-free high quality $2 \mu$ m-thick $\mathrm{A} 10.5 \mathrm{Ga} 0.5 \mathrm{~N}$ grown on a $\mathrm{Si}$ substrate with a superlattice transition layer, Cryst. Eng. Comm. 22 (2020) 1160, https: //doi.org/10.1039/C9CE01677E

103. S. Zhao et al., Molecular beam epitaxy growth of Alrich $\mathrm{AlGaN}$ nanowires for deep ultraviolet optoelectronics, APL Mater. 4 (2016), https://doi.org/10.1063/1. 4961680

104. H. Sun et al., Surface-Passivated AlGaN Nanowires for Enhanced Luminescence of Ultraviolet Light Emitting Diodes, ACS Photonics 5 (2018) 964, https://doi.org/10. 1021/acsphotonics.7b01235

105. S. M. Sadaf, Y. H. Ra, S. Zhao, T. Szkopek, and Z. Mi, Structural and electrical characterization of monolithic core-double shell $\mathrm{n}-\mathrm{GaN} / \mathrm{Al} / \mathrm{p}-\mathrm{AlGaN}$ nanowire heterostructures grown by molecular beam epitaxy, Nanoscale 11 (2019) 408, https: //doi.org/10.1039/c9nr00081j

106. R. F. Pierret, Advanced Semiconductor Fundamentals, Prentice Hall (2003).

107. J. J. Huang, H. C. Kuo, and S. C. Shen, Nitride Semiconductor Light-Emitting Diodes (LEDs): Materials, Technologies and Applications, Elsevier Science (2017), https : / / doi .org/ 10.1016/C2016-0-01551-6

108. C. R. Haughn et al., Highly radiative nature of ultra-thin cplane Al-rich AlGaN/AlN quantum wells for deep ultraviolet emitters, Appl. Phys. Lett. 114 (2019) 102101, https: //doi.org/10.1063/1.5087543

109. Q. Guo et al., The polarization field in Al-rich AlGaN multiple quantum wells, Jpn. J. Appl. Phys. 58 (2019), https: //doi.org/10.7567/1347-4065/ab07a9
110. N. Grandjean, J. Massies, and M. Leroux, Self-limitation of $\mathrm{AlGaN} / \mathrm{GaN}$ quantum well energy by built-in polarization field, Appl. Phys. Lett. 74 (1999) 2361, https://doi.org/10. $1063 / 1.123851$

111. N. Grandjean, B. Damilano, S. Dalmasso, M. Leroux, M. Laügt, and J. Massies, Built-in electric-field effects in wurtzite AlGaN/GaN quantum wells, J. Appl. Phys. 86 (1999) 3714, https://doi.org/10.1063/1.371241

112. M. A. Khan et al., $13 \mathrm{~mW}$ operation of a 295-310 nm AlGaN UV-B LED with a p-AlGaN transparent contact layer for real world applications. J. Mater. Chem. C 7 (2019) 143, https://doi.org/10.1039/C8TC03825B

113. N. Norimichi, H. Hirayama, T. Yatabe, and N. Kamata, 222 $\mathrm{nm}$ single-peaked deep-UV LED with thin AlGaN quantum well layers, Phys. status solidi c 6 (2009) S459, https: //doi.org/10.1002/pssc.200880923

114. H. Hirayama, N. Noguchi, T. Yatabe, and N. Kamata, 227 nm AlGaN Light-Emitting Diode with $0.15 \mathrm{~mW}$ Output Power Realized using a Thin Quantum Well and AlN Buffer with Reduced Threading Dislocation Density, Appl. Phys. Express 1 (2008) 51101, https : / /doi.org/10.1143/apex.1. 051101

115. U. Köhler et al., Optical Characterization of Cubic AlGaN/GaN Quantum Wells, Phys. status solidi 192 (2002)129, https://doi.org/10.1002/1521-396X(200207) 192:1〈129::AID-PSSA129〉3.0.CO;2-K

116. A. Islam, D.-S. Shim, and J.-I. Shim, Enhanced Radiative Recombination Rate by Local Potential Fluctuation in InGaN/AlGaN Near-Ultraviolet Light-Emitting Diodes, Appl. Sci. 9 (2019) 871, https://doi.org/10.3390/ app9050871

117. S. M. Islam et al., MBE-grown 232-270 nm deep-UV LEDs using monolayer thin binary GaN/AlN quantum heterostructures, Appl. Phys. Lett. 110 (2017), https://doi.org/ $10.1063 / 1.4975068$

118. D. Liu et al., $226 \mathrm{~nm} \mathrm{AlGaN/AlN} \mathrm{UV} \mathrm{LEDs} \mathrm{using} \mathrm{p-type}$ Si for hole injection and UV reflection, Appl. Phys. Lett. 113 (2018), https://doi.org/10.1063/1.5038044

119. Y. Kashima et al., High external quantum efficiency (10\%) AlGaN-based deep-ultraviolet light-emitting diodes achieved by using highly reflective photonic crystal on p-AlGaN contact layer, Appl. Phys. Express 11 (2018) 1, https : //doi. org/10.7567/APEX.11.012101

120. T. Takano et al., Deep-ultraviolet light-emitting diodes with external quantum efficiency higher than $20 \%$ at $275 \mathrm{~nm}$ achieved by improving light-extraction efficiency, Appl. Phys. Express 10 (2017), https: / /doi.org/10.7567/APEX. 10.031002 .

121. H. Hirayama, N. Maeda, S. Fujikawa, S. Toyoda, and N. Kamata, Recent progress and future prospects of $\mathrm{AlGaN}$ - diodes, Jpn. J. Appl. Phys. 53 (2014) 100209, https://doi.org/ $10.7567 /$ JJAP . 53.100209

122. M. Kaneda et al., Uneven AlGaN multiple quantum well for deep-ultraviolet LEDs grown on macrosteps and impact on electroluminescence spectral output, Jpn. J. Appl. Phys. 56 (2017) 61002, https://doi.org/10.7567/jjap.56. 061002 
123. M. Usman, A.-R. Anwar, and M. Munsif, Review-A Survey of Simulations on Device Engineering of GaNBased Light-Emitting Diodes, ECS J. Solid State Sci. Technol. 9 (2020) 66002, https://doi.org/10.1149/ 2162-8777/aba1cc

124. M. Shatalov, R. Jain, T. Saxena, A. Dobrinsky, and M. Shur, Chapter Two - Development of Deep UV LEDs and Current Problems in Material and Device Technology., in III-Nitride Semicond. Optoelectron., Z. Mi and C. B. T.-S. and S. Jagadish, Eds., Elsevier (2017) 45, https://doi.org/10.1016/ bs.semsem.2016.08.002

125. M. A. Khan et al., External Quantum Efficiency of $6.5 \%$ at $300 \mathrm{~nm}$ Emission and $4.7 \%$ at $310 \mathrm{~nm}$ Emission on Bare Wafer of AlGaN-Based UVB LEDs, ACS Appl. Electron. Mater. 2 (2020)1892, https: //doi.org/10.1021/acsaelm. $0 c 00172$

126. J. P. Liu et al., Barrier effect on hole transport and carrier distribution in InGaN/GaN multiple quantum well visible lightemitting diodes, Appl. Phys. Lett. 93(2008)21102, https: //doi.org/10.1063/1.2957667

127. M. Ajmal Khan, E. Matsuura, Y. Kashima, and H. Hirayama, Influence of Undoped-AlGaN Final Barrier of MQWs on the Performance of Lateral-Type UVB LEDs, Phys. status solidi 216(2019)1970059, https://doi.org/10.1002/ pssa.201970059

128. Z.-H. Zhang et al., Nearly Efficiency-Droop-Free AlGaNBased Ultraviolet Light-Emitting Diodes with a Specifically Designed Superlattice p-Type Electron Blocking Layer for High $\mathrm{Mg}$ Doping Efficiency, Nanoscale Res. Lett. 13 (2018) 122, https://doi.org/10.1186/ s11671-018-2539-9.

129. S. Wang, Y. A. Yin, H. Gu, N. Wang, and L. Liu, Graded AlGaN/AlGaN Superlattice Insert Layer Improved Performance of AlGaN-Based Deep Ultraviolet Light-Emitting Diodes, $J$. Disp. Technol. 12 (2016) 1112, https://doi.org/10. 1109/JDT.2016.2583438

130. J. Huang et al., Study of Deep Ultraviolet Light-Emitting Diodes with a p-AlInN/AlGaN Superlattice Electron-Blocking Layer, J. Electron. Mater. 46 (2017) 4527, https://doi. org/10.1007/s11664-017-5413-0

131. X. Fan et al., Efficiency improvements in AlGaN-based deep ultraviolet light-emitting diodes using inverted-V-shaped graded Al composition electron blocking layer, Superlattices Microstruct. 88 (2015) 467, https: //doi.org/10. $1016 / j$.spmi.2015.10.003

132. Z.-H. Zhang et al., Increasing the hole energy by grading the alloy composition of the p-type electron blocking layer for very high-performance deep ultraviolet light-emitting diodes, Photon. Res. 7 (2019), https://doi.org/10.1364/PRJ. $7.0000 \mathrm{~B} 1$

133. M. Katsuragawa et al., Thermal ionization energy of Si and $\mathrm{Mg}$ in AlGaN, J. Cryst. Growth 189-190 (1998) 528, https : //doi.org/10.1016/S0022-0248(98)00345-5.

134. B. Sarkar et al., N- and P- type Doping in Al-rich AlGaN and AlN, ECS Trans. 86 (2018) 25, https://doi.org/10. $1149 / 08612.0025$ ecst
135. A. T. M. Golam Sarwar, B. J. May, M. F. Chisholm, G. J. Duscher, and R. C. Myers, Ultrathin GaN quantum disk nanowire LEDs with sub-250 nm electroluminescence, Nanoscale 8 (2016) 8024, https: //doi.org/10.1039/ c6nr00132g

136. H. Hirayama et al., Realization of over $10 \% \mathrm{EQE}$ AlGaN deep-UV LED by using transparent p-AlGaN contact layer, Conf. Dig. - IEEE Int. Semicond. Laser Conf. 91 (2016) 7.

137. S. Bharadwaj et al., High-temperature p-type polarization doped AlGaN cladding for sub-250 nm deep-UV quantum well LEDs by MBE, Device Res. Conf. - Conf. Dig. DRC 10 (2017) 5, https: //doi.org/10.1109/DRC.2017. 7999517

138. C. Kuhn, L. Sulmoni, M. Guttmann, J. Glaab, N. Susilo, T. Wernicke, M. Weyers, and M. Kneissl, MOVPE-grown AlGaNbased tunnel heterojunctions enabling fully transparent UVC LEDs, Photonics Res. 7(2019), https://doi.org/10. $1364 / \mathrm{prj} .7 .0000 \mathrm{~b} 7$

139. C. A. Hernández-Gutierrez et al., A new method of making ohmic contacts to p-GaN, Nucl. Instruments Methods Phys. Res. Sect. B Beam Interact. with Mater. Atoms 388 (2016) 35, https://doi.org/10.1016/j.nimb.2016.11. 001.

140. T. Oto, R. G. Banal, K. Kataoka, M. Funato, and Y. Kawakami, $100 \mathrm{~mW}$ deep-ultraviolet emission from aluminium-nitride-based quantum wells pumped by an electron beam, Nat. Photonics 4 (2010) 767, https://doi.org/ $10.1038 /$ nphoton.2010.220

141. K. Ding, V. Avrutin, Ü. Özgür, and H. Morkoç, Status of growth of group III-nitride heterostructures for deep ultraviolet light-emitting diodes, Crystals 7 (2017), https: / / doi. org/10.3390/cryst 7100300

142. L. Lu, G. G. Ding, Y. Zhang, Y. H. Liu, and F. J. Xu, Improved performance of AlGaN-based deep ultraviolet light-emitting diode using modulated-taper design for p-AlGaN layer, Semicond. Sci. Technol. 33 (2018) 35008, https://doi.org/ $10.1088 / 1361-6641 / \mathrm{aaa} 8 \mathrm{~b} 2$

143. J.-W. Min et al., Unleashing the potential of molecular beam epitaxy grown AlGaN-based ultraviolet-spectrum nanowires devices, J. Nanophotonics 12 (2018), https://doi.org/ $10.1117 / 1$.jnp.12.043511

144. Z. Chen, J. Hoo, Y. Chen, V. Wang, and S. Guo, Study of aln based materials grown on nano-patterned sapphire substrates for deep ultraviolet led applications, Jpn. J. Appl. Phys. 58 (2019), https://doi.org/10.7567/1347-4065/ ab09de.

145. W. Luo et al., Enhanced p-type conduction in AlGaN grown by metal-source flow-rate modulation epitaxy, Appl. Phys. Lett. 113 (2018), https: //doi.org/10.1063/1.5040334

146. C. A. Hernández-Gutiérrez et al., Study of the heavily p-type doping of cubic GaN with Mg, Sci. Rep. 10 (2020) 1, https : //doi.org/10.1038/s41598-020-73872-w

147. Z. Wu et al., High hole concentration in nonpolar a-plane p-AlGaN films with Mg-delta doping technique, Superlattices Microstruct. 109 (2017) 880, https : / / doi .org/10. $1016 / j . s p m i .2017 .06 .008$ 
148. Q. Si, H. Chen, S. Li, S. Lu, and J. Kang, Improved characteristics of AlGaN-based deep ultraviolet light-emitting diodes with superlattice p-type doping, IEEE Photonics J. 9 (2017) 1, https://doi.org/10.1109/JPHOT.2017. 2699322

149. M. Liu et al., An InGaN/GaN Superlattice to Enhance the Performance of Green LEDs: Exploring the Role of V-Pits, Nanomaterials 8 (2018) 450, https://doi.org/10.3390/ nano8070450

150. B. P. Gunning et al., Comprehensive study of the electronic and optical behavior of highly degenerate p-type Mg-doped GaN and AlGaN, J. Appl. Phys. 117 (2015) 20, https:// doi.org/10.1063/1.4906464

151. T. C. Zheng et al., Improved p-type conductivity in Al-rich AlGaN using multidimensional Mg-doped superlattices, Sci. Rep. 6 (2016) 1, https://doi.org/10.1038/srep21897

152. R. K. Mondal, V. Chatterjee, and S. Pal, AlInGaN-based superlattice p-region for improvement of performance of deep UV LEDs, Opt. Mater. (Amst). 104 (2020) 109846, https : //doi.org/10.1016/j.optmat.2020.109846

153. L. Y. Wang et al., Efficiency enhancement of ultraviolet light-emitting diodes with segmentally graded p-type AlGaN layer, Chinese Phys. B 28 (2019), https ://doi.org/10. 1088/1674-1056/28/1/018503

154. Z. Ren et al., III-Nitride Deep UV LED Without Electron Blocking Layer, IEEE Photonics J. 11 (2019), https:// doi.org/10.1109/JPHOT.2019.2902125.

155. Y. Hou, and Z. Guo, Improve the electrical and optical performance of deep ultraviolet light-emitting diodes with a w-shaped p-AlGaN layer, J. Mater. Sci. Mater. Electron. (2019), https: //doi.org/10.1007/s10854-019-01051-3

156. G. Li et al., Performance improvement of UV light-emitting diodes with triangular quantum barriers, IEEE Photonics Technol. Lett. 30 (2018) 1071, https://doi.org/10.1109/ LPT.2018.2827371

157. M. Z. Kauser, A. Osinsky, A. M. Dabiran, and P. P. Chow, Enhanced vertical transport in p-type $\mathrm{AlGaN} / \mathrm{GaN}$ superlattices, Appl. Phys. Lett. 85 (2004) 5275, https ://doi.org/10. $1063 / 1.1828230$

158. P. Pampili, and P. J. Parbrook, Doping of III-nitride materials, Mater. Sci. Semicond. Process. 62 (2017) 180, https: //doi.org/10.1016/j.mssp.2016.11.006

159. E. F. Schubert, Delta-Doping of Semiconductors: Electronic, Optical, and Structural Properties of Materials and Devices, (1994) https://doi.org/10.1016/ S0080-8784(08)62662-9.

160. S. Yang et al., Influence of Silicon-Doping in n-AlGaN Layer on the Optical and Electrical Performance of Deep Ultraviolet Light-Emitting Diodes. Russ. J. Phys. Chem. A 93 (2019) 2817, https://doi.org/10.1134/S003602441913034X

161. G. Greco, F. Iucolano, and F. Roccaforte, Ohmic contacts to Gallium Nitride materials, Appl. Surf. Sci. 383 (2016) 324, https://doi.org/10.1016/j.apsusc.2016. 04.016 .
162. A. Knauer et al., High power uvb light emitting diodes with optimized n-algan contact layers, Jpn. J. Appl. Phys. 58 (2019), https://doi.org/10.7567/1347-4065/ab0f13.

163. K. Mori et al., Low-ohmic-contact-resistance V-based electrode for n-type AlGaN with high AlN molar fraction, Jpn. J. Appl. Phys. 55 (2016), https://doi.org/10.7567/ JJAP. 55.05FL03

164. M. Hiroki, and K. Kumakura, Ohmic contact to AlN:Si using graded AlGaN contact layer, Appl. Phys. Lett. 115 (2019), https://doi.org/10.1063/1.5124936

165. M. Jo, N. Maeda, and H. Hirayama, Enhanced light extraction in 260nm light-emitting diode with a highly transparent p-AlGaN layer, Appl. Phys. Express 9 (2016) 7, https : //doi.org/10.7567/APEX.9.012102.

166. G. B. Fayisa et al., Enhanced light extraction efficiency of micro-ring array $\mathrm{AlGaN}$ deep ultraviolet light-emitting diodes, Jpn. J. Appl. Phys. 56(2017), https://doi.org/10. $7567 /$ JJAP. 56.092101

167. X. Chen, K.-Y. Ho, and Y.-R. Wu, Modeling and optimization of $\mathrm{p}$-AlGaN super lattice structure as the p-contact and transparent layer in AlGaN UVLEDs, Opt. Express 23 (2015) 32367, https://doi.org/10.1364/oe.23.032367

168. H. K. Cho et al., Highly Reflective p-Contacts Made of PdAl on Deep Ultraviolet Light-Emitting Diodes, IEEE Photonics Technol. Lett. 29 (2017) 2222, https : // doi.org/10. $1109 / \mathrm{LPT} .2017 .2771526$

169. Zheng-Fei Hu, X.-Y. Li, and Y. Zhang, High Resolution Investigation on the NiAu Ohmic Contact to p-AlGaN-GaN Heterostructure, Phys. Solid State 61 (2019) 2295, https: //doi.org/10.1134/s1063783419120151

170. Z. F. Hu, X. Y. Li, and Y. Zhang, Characteristics of $\mathrm{Ni} / \mathrm{Au} / \mathrm{Ni} / \mathrm{Au}$ ohmic contact in a $\mathrm{p}-\mathrm{AlGaN} / \mathrm{GaN}$ semiconductor, IOP Conf. Ser. Mater. Sci. Eng. 770 (2020), https: //doi.org/10.1088/1757-899X/770/1/012018

171. T. Passow, et al., Ni/Ag as low resistive ohmic contact to ptype AlGaN for UV LEDs., in Light. Diodes Mater. Devices, Appl. Solid State Light. (2010) 190, https://doi.org/ $10.1117 / 12.841968$

172. M. A. Bergmann et al., Electrochemical etching of AlGaN for the realization of thin-film devices, Appl. Phys. Lett. 115 (2019) 182103, https://doi.org/10.1063/1.5120397

173. S. Yasue et al., The dependence of AlN molar fraction of Al$\mathrm{GaN}$ in wet etching by using tetramethylammonium hydroxide aqueous solution, Jpn. J. Appl. Phys. 58 (2019), https: //doi.org/10.7567/1347-4065/ab112a

174. S. Banna et al., Pulsed high-density plasmas for advanced dry etching processes, J. Vac. Sci. Technol. A 30 (2012) 40801, https://doi.org/10.1116/1.4716176

175. Y. J. Sung et al., Light extraction enhancement of AlGaNbased vertical type deep-ultraviolet light-emitting-diodes by using highly reflective ITO/Al electrode and surface roughening, Opt. Express 27 (2019) 29930, https://doi.org/ $10.1364 / \mathrm{OE} .27 .029930$

176. S. J. Fonash, Chapter Three - Structures, Materials, and Scale, (2010) 67, https://doi.org/10.1016/ B978-0-12-374774-7.00003-0. 
177. W. S. Leong et al., Low Resistance Metal Contacts to MoS2 Devices with Nickel-Etched-Graphene Electrodes, ACS Nano 9 (2015) 869, https : //doi.org/10.1021/nn506567r

178. N. Maeda, M. Jo, and H. Hirayama, Improving the Efficiency of AlGaN Deep-UV LEDs by Using Highly Reflective Ni/Al p-Type Electrodes, Phys. Status Solidi Appl. Mater. Sci. (2018) 1, https://doi.org/10.1002/pssa.201700435.

179. K. H. Kim, T. H. Lee, K. R. Son, and T. G. Kim, Performance improvements in AlGaN-based ultraviolet light-emitting diodes due to electrical doping effects, Mater. Des. 153 (2018) 94, https://doi.org/10.1016/j.matdes. 2018.04 .086

180. S.-Y. Lee et al., Heavy $\mathrm{Mg}$ Doping to Form Reliable $\mathrm{Rh}$ Reflective Ohmic Contact for $278 \mathrm{~nm}$ Deep Ultraviolet
AlGaN-Based Light-Emitting Diodes, ECS J. Solid State Sci. Technol. 9 (2020) 065016, https://doi.org/10.1149/ 2162-8777/aba914

181. J. Lee, T.-Y. Seong, and H. Amano, Oblique-Angle Deposited $\mathrm{SiO} / \mathrm{Al}$ Omnidirectional Reflector for Enhancing the Performance of AlGaN-Based Ultraviolet Light-Emitting Diode, ECS J. Solid State Sci. Technol. 9 (2020) 26005, https : / / doi . org/10.1149/2162-8777/ab709a

182. R. K. Mondal, V. Chatterjee, and S. Pal, Efficient Carrier Transport for AlGaN-Based Deep-UV LEDs with Graded Superlattice p-AlGaN, IEEE Trans. Electron Devices 67 (2020) 1674, https://doi.org/10.1109/TED.2020. 2974408 